\title{
An invitation to higher gauge theory
}

\author{
John C. Baez • John Huerta
}

Received: 20 March 2010 / Accepted: 31 July 2010 / Published online: 19 August 2010

(C) The Author(s) 2010. This article is published with open access at Springerlink.com

\begin{abstract}
In this easy introduction to higher gauge theory, we describe parallel transport for particles and strings in terms of 2-connections on 2-bundles. Just as ordinary gauge theory involves a gauge group, this generalization involves a gauge '2-group'. We focus on 6 examples. First, every abelian Lie group gives a Lie 2-group; the case of $U(1)$ yields the theory of U(1) gerbes, which play an important role in string theory and multisymplectic geometry. Second, every group representation gives a Lie 2-group; the representation of the Lorentz group on 4d Minkowski spacetime gives the Poincaré 2-group, which leads to a spin foam model for Minkowski spacetime. Third, taking the adjoint representation of any Lie group on its own Lie algebra gives a 'tangent 2-group', which serves as a gauge 2-group in $4 \mathrm{~d} B F$ theory, which has topological gravity as a special case. Fourth, every Lie group has an 'inner automorphism 2-group', which serves as the gauge group in $4 \mathrm{~d} B F$ theory with cosmological constant term. Fifth, every Lie group has an 'automorphism 2-group', which plays an important role in the theory of nonabelian gerbes. And sixth, every compact simple Lie group gives a 'string 2-group'. We also touch upon higher structures such as the 'gravity 3-group', and the Lie 3-superalgebra that governs 11-dimensional supergravity.
\end{abstract}

Keywords Category $\cdot$ Gerbe $\cdot$ Higher gauge theory $\cdot$ String $\cdot$ 2-category 2-group

\footnotetext{
J. C. Baez $(\varangle) \cdot$ J. Huerta

Department of Mathematics, University of California,

Riverside, CA 92521, USA

e-mail: baez@math.ucr.edu

J. Huerta

e-mail: huerta@math.ucr.edu
} 


\section{Introduction}

Higher gauge theory is a generalization of gauge theory that describes parallel transport, not just for point particles, but also for higher-dimensional extended objects. It is a beautiful new branch of mathematics, with a lot of room left for exploration. It has already been applied to string theory and loop quantum gravity-or more specifically, spin foam models. This should not be surprising, since while these rival approaches to quantum gravity disagree about almost everything, they both agree that point particles are not enough: we need higher-dimensional extended objects to build a theory sufficiently rich to describe the quantum geometry of spacetime. Indeed, many existing ideas from string theory and supergravity have recently been clarified by higher gauge theory $[82,83]$. But we may also hope for applications of higher gauge theory to other less speculative branches of physics, such as condensed matter physics.

Of course, for this to happen, more physicists need to learn higher gauge theory. It would be great to have a comprehensive introduction to the subject which started from scratch and led the reader to the frontiers of knowledge. Unfortunately, mathematical work in this subject uses a wide array of tools, such as $n$-categories, stacks, gerbes, Deligne cohomology, $L_{\infty}$-algebras, Kan complexes, and $(\infty, 1)$-categories, to name just a few. While these tools are beautiful, important in their own right, and perhaps necessary for a deep understanding of higher gauge theory, learning them takes time-and explaining them all would be a major project.

Our goal here is far more modest. We shall sketch how to generalize the theory of parallel transport from point particles to 1-dimensional objects, such as strings. We shall do this starting with a bare minimum of prerequisites: manifolds, differential forms, Lie groups, Lie algebras, and the traditional theory of parallel transport in terms of bundles and connections. We shall give a small taste of the applications to physics, and point the reader to the literature for more details.

In Sect. 2 we start by explaining categories, functors, and how parallel transport for particles can be seen as a functor taking any path in a manifold to the operation of parallel transport along that path. In Sect. 3 we 'add one' and explain how parallel transport for particles and strings can be seen as '2-functor' between '2-categories'. This requires that we generalize Lie groups to 'Lie 2-groups'. In Sect. 4 we describe many examples of Lie 2-groups, and sketch some of their applications:

- Section 4.1: shifted abelian groups, U(1) gerbes, and their role in string theory and multisymplectic geometry.

- Section 4.2: the Poincaré 2-group and the spin foam model for 4d Minkowski spacetime.

- Section 4.3: tangent 2-groups, $4 \mathrm{~d} B F$ theory and topological gravity.

- Section 4.4: inner automorphism 2-groups and 4d BF theory with cosmological constant term.

- Section 4.5: automorphism 2-groups, nonabelian gerbes, and the gravity 3-group.

- Section 4.6: string 2-groups, string structures, the passage from Lie $n$-algebras to Lie $n$-groups, and the Lie 3-superalgebra governing 11-dimensional supergravity.

Finally, in Sect. 5 we discuss gauge transformations, curvature and nontrivial 2-bundles. 


\section{Categories and connections}

A category consists of objects, which we draw as dots:

and morphisms between objects, which we draw as arrows between dots:

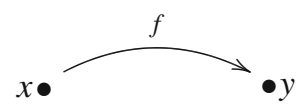

You should think of objects as 'things' and morphisms as 'processes'. The main thing you can do in a category is take a morphism from $x$ to $y$ and a morphism from $y$ to $z$ :

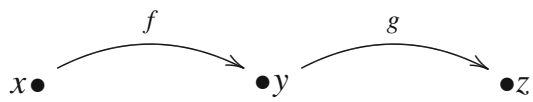

and 'compose' them to get a morphism from $x$ to $z$ :

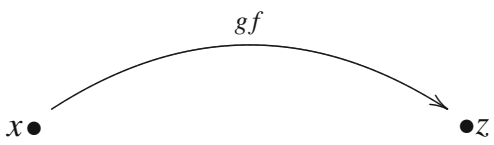

The most famous example is the category Set, which has sets as objects and functions as morphisms. Most of us know how to compose functions, and we have a pretty good intuition of how this works. So, it can be helpful to think of morphisms as being like functions. But as we shall soon see, there are some very important categories where the morphisms are not functions.

Let us give the formal definition. A category consists of:

- A collection of objects, and

- for any pair of objects $x, y$, a set of morphisms $f: x \rightarrow y$. Given a morphism $f: x \rightarrow y$, we call $x$ its source and $y$ its target.

- Given two morphisms $f: x \rightarrow y$ and $g: y \rightarrow z$, there is a composite morphism $g f: x \rightarrow z$. Composition satisfies the associative law:

$$
(h g) f=h(g f) \text {. }
$$

- For any object $x$, there is an identity morphism $1_{x}: x \rightarrow x$. These identity morphisms satisfy the left and right unit laws:

$$
1_{y} f=f=f 1_{x}
$$

for any morphism $f: x \rightarrow y$.

The hardest thing about category theory is getting your arrows to point the right way. It is standard in mathematics to use $f g$ to denote the result of doing first $g$ and then $f$. 
In pictures, this backwards convention can be annoying. But rather than trying to fight it, let us give in and draw a morphism $f: x \rightarrow y$ as an arrow from right to left:

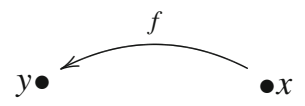

Then composition looks a bit better:

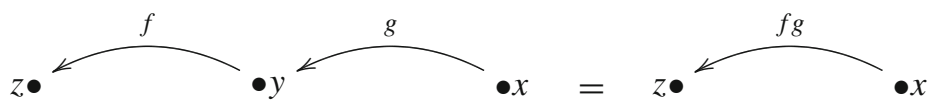

An important example of a category is the 'path groupoid' of a space $X$. We give the precise definition below, but the basic idea is to take the diagrams we have been drawing seriously! The objects are points in $X$, and morphisms are paths:

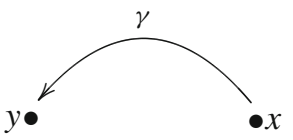

We also get examples from groups. A group is the same as a category with one object where all the morphisms are invertible. The morphisms of this category are the elements of the group. The object is there just to provide them with a source and target. We compose the morphisms using the multiplication in the group.

In both these examples, a morphism $f: x \rightarrow y$ is not a function from $x$ to $y$. And these two examples have something else in common: they are important in gauge theory! We can use a path groupoid to describe the possible motions of a particle through spacetime. We can use a group to describe the symmetries of a particle. And when we combine these two examples, we get the concept of connection-the basic field in any gauge theory.

How do we combine these examples? We do it using a map between categories. A map between categories is called a 'functor'. A functor from a path groupoid to a group will send every object of the path groupoid to the same object of our group. After all, a group, regarded as a category, has only one object. But this functor will also send any morphism in our path groupoid to a group element. In other words, it will assign a group element to each path in our space. This group element describes how a particle transforms as it moves along that path.

But this is precisely what a connection does! A connection lets us compute for any path a group element describing parallel transport along that path. So, the language of categories and functors quickly leads us to the concept of connection-but with an emphasis on parallel transport.

The following theorem makes these ideas precise. Let us first state the theorem, then define the terms involved, and then give some idea of how it is proved:

Theorem 1 For any Lie group $G$ and any smooth manifold $M$, there is a one-to-one correspondence between: 
1. connections on the trivial principal $G$-bundle over $M$,

2. $\mathfrak{g}$-valued 1-forms on $M$, where $\mathfrak{g}$ is the Lie algebra of $G$, and

3. smooth functors

$$
\text { hol: } \mathcal{P}_{1}(M) \rightarrow G
$$

where $\mathcal{P}_{1}(M)$ is the path groupoid of $M$.

We assume you are familiar with the first two items. Our goal is to explain the third. We must start by explaining the path groupoid.

Suppose $M$ is a manifold. Then the path groupoid $\mathcal{P}_{1}(M)$ is roughly a category in which objects are points of $M$ and a morphism from $x$ to $y$ is a path from $x$ to $y$. We compose paths by gluing them end to end. So, given a path $\delta$ from $x$ to $y$, and a path $\gamma$ from $y$ to $z$ :

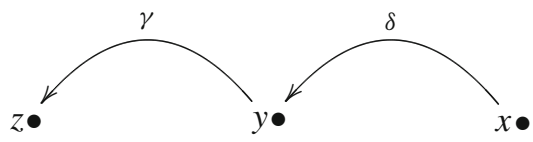

we would like $\gamma \delta$ to be the path from $x$ to $z$ built from $\delta$ and $\gamma$.

However, we need to be careful about the details to make sure that the composite path $\gamma \delta$ is well-defined, and that composition is associative! Since we are studying paths in a smooth manifold, we want them to be smooth. But the path $\gamma \delta$ may not be smooth: there could be a 'kink' at the point $y$.

There are different ways to get around this problem. One is to work with piecewise smooth paths. But here is another approach: say that a path

$$
\gamma:[0,1] \rightarrow M
$$

is lazy if it is smooth and also constant in a neighborhood of $t=0$ and $t=1$. The idea is that a lazy hiker takes a rest before starting a hike, and also after completing it. Suppose $\gamma$ and $\delta$ are smooth paths and $\gamma$ starts where $\delta$ ends. Then we define their composite

$$
\gamma \delta:[0,1] \rightarrow M
$$

in the usual way:

$$
(\gamma \delta)(t)=\left\{\begin{array}{cc}
\delta(2 t) & \text { if } 0 \leq t \leq \frac{1}{2} \\
\gamma(2 t-1) & \text { if } \frac{1}{2} \leq t \leq 1
\end{array}\right.
$$

In other words, $\gamma \delta$ spends the first half of its time moving along $\delta$, and the second half moving along $\gamma$. In general the path $\gamma \delta$ may not be smooth at $t=\frac{1}{2}$. However, if $\gamma$ and $\delta$ are lazy, then their composite is smooth — and it, too, is lazy! 
So, lazy paths are closed under composition. Unfortunately, composition of lazy paths is not associative. The paths $(\alpha \beta) \gamma$ and $\alpha(\beta \gamma)$ differ by a smooth reparametrization, but they are not equal. To solve this problem, we can take certain equivalence classes of lazy paths as morphisms in the path groupoid.

We might try 'homotopy classes' of paths. Remember, a homotopy is a way of interpolating between paths:

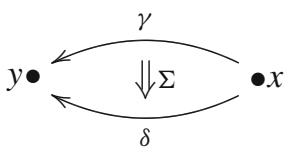

More precisely, a homotopy from the path $\gamma:[0,1] \rightarrow M$ to the path $\delta:[0,1] \rightarrow M$ is a smooth map

$$
\Sigma:[0,1]^{2} \rightarrow M
$$

such that $\Sigma(0, t)=\gamma(t)$ and $\Sigma(1, t)=\delta(t)$. We say two paths are homotopic, or lie in the same homotopy class, if there is a homotopy between them.

There is a well-defined category where the morphisms are homotopy classes of lazy paths. Unfortunately this is not right for gauge theory, since for most connections, parallel transport along homotopic paths gives different results. In fact, parallel transport gives the same result for all homotopic paths if and only if the connection is flat.

So, unless we are willing to settle for flat connections, we need a more delicate equivalence relation between paths. Here the concept of 'thin' homotopy comes to our rescue. A homotopy is thin if it sweeps out a surface that has zero area. In other words, it is a homotopy $\Sigma$ such that the rank of the differential $d \Sigma$ is less than 2 at every point. If two paths differ by a smooth reparametrization, they are thinly homotopic. But there are other examples, too. For example, suppose we have a path $\gamma: x \rightarrow y$, and let $\gamma^{-1}: y \rightarrow x$ be the reverse path, defined as follows:

$$
\gamma^{-1}(t)=\gamma(1-t)
$$

Then the composite path $\gamma^{-1} \gamma$, which goes from $x$ to itself:

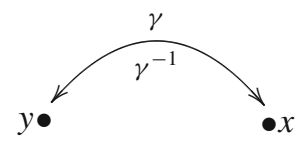

is thinly homotopic to the constant path that sits at $x$. The reason is that we can shrink $\gamma^{-1} \gamma$ down to the constant path without sweeping out any area.

We define the path groupoid $\mathcal{P}_{1}(M)$ to be the category where:

- Objects are points of $M$.

- Morphisms are thin homotopy classes of lazy paths in $M$. 
- If we write $[\gamma]$ to denote the thin homotopy class of the path $\gamma$, composition is defined by

$$
[\gamma][\delta]=[\gamma \delta]
$$

- For any point $x \in M$, the identity $1_{x}$ is the thin homotopy class of the constant path at $x$.

With these rules, it is easy to check that $\mathcal{P}_{1}(M)$ is a category. The most important point is that since the composite paths $(\alpha \beta) \gamma$ and $\alpha(\beta \gamma)$ differ by a smooth reparametrization, they are thinly homotopic. This gives the associative law when we work with thin homotopy classes.

But as its name suggests, $\mathcal{P}_{1}(M)$ is better than a mere category. It is a groupoid: that is, a category where every morphism $\gamma: x \rightarrow y$ has an inverse $\gamma^{-1}: y \rightarrow x$ satisfying

$$
\gamma^{-1} \gamma=1_{x} \text { and } \gamma \gamma^{-1}=1_{y}
$$

In $\mathcal{P}_{1}(M)$, the inverse is defined using the concept of a reverse path:

$$
[\gamma]^{-1}=\left[\gamma^{-1}\right]
$$

The rules for an inverse only hold in $\mathcal{P}_{1}(M)$ after we take thin homotopy classes. After all, the composites $\gamma \gamma^{-1}$ and $\gamma^{-1} \gamma$ are not constant paths, but they are thinly homotopic to constant paths. But henceforth, we will relax and write simply $\gamma$ for the morphism in the path groupoid corresponding to a path $\gamma$, instead of $[\gamma]$.

As the name suggests, groupoids are a bit like groups. Indeed, a group is secretly the same as a groupoid with one object! In other words, suppose we have group $G$. Then there is a category where:

- There is only one object, $\bullet$.

- Morphisms from $\bullet$ to $\bullet$ are elements of $G$.

- Composition of morphisms is multiplication in the group $G$.

- The identity morphism 1 • is the identity element of $G$.

This category is a groupoid, since every group element has an inverse. Conversely, any groupoid with one object gives a group. Henceforth we will freely switch back and forth between thinking of a group in the traditional way, and thinking of it as a one-object groupoid.

How can we use groupoids to describe connections? It should not be surprising that we can do this, now that we have our path groupoid $\mathcal{P}_{1}(M)$ and our one-object groupoid $G$ in hand. A connection gives a map from $\mathcal{P}_{1}(M)$ to $G$, which says how to transform a particle when we move it along a path. More precisely: if $G$ is a Lie group, any connection on the trivial $G$-bundle over $M$ yields a map, called the parallel transport map or holonomy, that assigns an element of $G$ to each path:

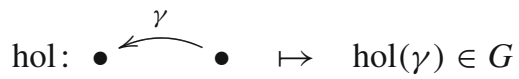


In physics notation, the holonomy is defined as the path-ordered exponential of some $\mathfrak{g}$-valued 1-form $A$, where $\mathfrak{g}$ is the Lie algebra of $G$ :

$$
\operatorname{hol}(\gamma)=\mathcal{P} \exp \left(\int_{\gamma} A\right) \in G .
$$

The holonomy map satisfies certain rules, most of which are summarized in the word 'functor'. What is a functor? It is a map between categories that preserves all the structure in sight!

More precisely: given categories $C$ and $D$, a functor $F: C \rightarrow D$ consists of:

- a map $F$ sending objects in $C$ to objects in $D$, and

- another map, also called $F$, sending morphisms in $C$ to morphisms in $D$,

such that:

- given a morphism $f: x \rightarrow y$ in $C$, we have $F(f): F(x) \rightarrow F(y)$,

- $F$ preserves composition:

$$
F(f g)=F(f) F(g)
$$

when either side is well-defined, and

- $F$ preserves identities:

$$
F\left(1_{x}\right)=1_{F(x)}
$$

for every object $x$ of $C$.

The last property actually follows from the rest. The second to last-preserving composition-is the most important property of functors. As a test of your understanding, check that if $C$ and $D$ are just groups (that is, one-object groupoids) then a functor $F: C \rightarrow D$ is just a homomorphism.

Let us see what this definition says about a functor

$$
\text { hol: } \mathcal{P}_{1}(M) \rightarrow G
$$

where $G$ is some Lie group. This functor hol must send all the points of $M$ to the one object of $G$. More interestingly, it must send thin homotopy classes of paths in $M$ to elements of $G$ :

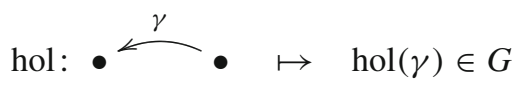

It must preserve composition:

$$
\operatorname{hol}(\gamma \delta)=\operatorname{hol}(\gamma) \operatorname{hol}(\delta)
$$


and identities:

$$
\operatorname{hol}\left(1_{x}\right)=1 \in G
$$

While they may be stated in unfamiliar language, these are actually well-known properties of connections! First, the holonomy of a connection along a path

$$
\operatorname{hol}(\gamma)=\mathcal{P} \exp \left(\int_{\gamma} A\right) \in G
$$

only depends on the thin homotopy class of $\gamma$. To see this, compute the variation of $\operatorname{hol}(\gamma)$ as we vary the path $\gamma$, and show the variation is zero if the homotopy is thin. Second, to compute the group element for a composite of paths, we just multiply the group elements for each one:

$$
\mathcal{P} \exp \left(\int_{\gamma \delta} A\right)=\mathcal{P} \exp \left(\int_{\gamma} A\right) \mathcal{P} \exp \left(\int_{\delta} A\right)
$$

And third, the path-ordered exponential along a constant path is just the identity:

$$
\mathcal{P} \exp \left(\int_{1_{x}} A\right)=1 \in G .
$$

All this information is neatly captured by saying hol is a functor. And Theorem 1 says this is almost all there is to being a connection. The only additional condition required is that hol be smooth. This means, roughly, that hol $(\gamma)$ depends smoothly on the path $\gamma$-more on that later. But if we drop this condition, we can generalize the concept of connection, and define a generalized connection on a smooth manifold $M$ to be a functor hol: $\mathcal{P}_{1}(M) \rightarrow G$.

Generalized connections have long played an important role in loop quantum gravity, first in the context of real-analytic manifolds [3], and later for smooth manifolds $[18,63]$. The reason is that if $M$ is any manifold and $G$ is a connected compact Lie group, there is a natural measure on the space of generalized connections. This means that you can define a Hilbert space of complex-valued square-integrable functions on the space of generalized connections. In loop quantum gravity these are used to describe quantum states before any constraints have been imposed. The switch from connections to generalized connections is crucial here-and the lack of smoothness gives loop quantum gravity its 'discrete' flavor.

But suppose we are interested in ordinary connections. Then we really want hol $(\gamma)$ to depend smoothly on the path $\gamma$. How can we make this precise?

One way is to use the theory of 'smooth groupoids' [17]. Any Lie group is a smooth groupoid, and so is the path groupoid of any smooth manifold. We can define smooth 
functors between smooth groupoids, and then smooth functors hol: $\mathcal{P}_{1}(M) \rightarrow G$ are in one-to-one correspondence with connections on the trivial principal $G$-bundle over $M$. We can go even further: there are more general maps between smooth groupoids, and maps hol: $\mathcal{P}_{1}(M) \rightarrow G$ of this more general sort correspond to connections on not necessarily trivial principal $G$-bundles over $M$. For details, see the work of Bartels [26], Schreiber and Waldorf [87].

But if this sounds like too much work, we can take the following shortcut. Suppose we have a smooth function $F:[0,1]^{n} \times[0,1] \rightarrow M$, which we think of as a parametrized family of paths. And suppose that for each fixed value of the parameter $s \in[0,1]^{n}$, the path $\gamma_{s}$ given by

$$
\gamma_{s}(t)=F(s, t)
$$

is lazy. Then our functor hol: $\mathcal{P}_{1}(M) \rightarrow G$ gives a function

$$
\begin{aligned}
{[0,1]^{n} } & \rightarrow G \\
s \quad & \mapsto \operatorname{hol}\left(\gamma_{s}\right) .
\end{aligned}
$$

If this function is smooth whenever $F$ has the above properties, then the functor hol: $\mathcal{P}_{1}(M) \rightarrow G$ is smooth.

Starting from this definition one can prove the following lemma, which lies at the heart of Theorem 1:

Lemma There is a one-to-one correspondence between smooth functors hol: $\mathcal{P}_{1}(M)$ $\rightarrow G$ and $\operatorname{Lie}(G)$-valued 1-forms $A$ on $M$.

The idea is that given a $\operatorname{Lie}(G)$-valued 1-form $A$ on $M$, we can define a holonomy for any smooth path as follows:

$$
\operatorname{hol}(\gamma)=\mathcal{P} \exp \left(\int_{\gamma} A\right),
$$

and then check that this defines a smooth functor hol: $\mathcal{P}_{1}(M) \rightarrow G$. Conversely, suppose we have a smooth functor hol of this sort. Then we can define hol $(\gamma)$ for smooth paths $\gamma$ that are not lazy, using the fact that every smooth path is thinly homotopic to a lazy one. We can even do this for paths $\gamma:[0, s] \rightarrow M$ where $s \neq 1$, since any such path can be reparametrized to give a path of the usual sort. Given a smooth path

$$
\gamma:[0,1] \rightarrow M
$$

we can truncate it to obtain a path $\gamma_{s}$ that goes along $\gamma$ until time $s$ :

$$
\gamma_{s}:[0, s] \rightarrow M
$$

By what we have said, $\operatorname{hol}\left(\gamma_{s}\right)$ is well-defined. Using the fact that hol: $\mathcal{P}_{1}(M) \rightarrow G$ is a smooth functor, one can check that hol $\left(\gamma_{s}\right)$ varies smoothly with $s$. So, we can 
differentiate it and define a $\operatorname{Lie}(G)$-valued 1-form $A$ as follows:

$$
A(v)=\left.\frac{d}{d s} \operatorname{hol}\left(\gamma_{s}\right)\right|_{s=0}
$$

where $v$ is any tangent vector at a point $x \in M$, and $\gamma$ is any smooth path with

$$
\gamma(0)=x, \quad \gamma^{\prime}(0)=v
$$

Of course, we need to check that $A$ is well-defined and smooth. We also need to check that if we start with a smooth functor hol, construct a 1-form $A$ in this way, and then turn $A$ back into a smooth functor, we wind up back where we started.

\section{2-Categories and 2-connections}

Now we want to climb up one dimension, and talk about '2-connections'. A connection tells us how particles transform as they move along paths. A 2-connection will also tell us how strings transform as they sweep out surfaces. To make this idea precise, we need to take everything we said in the previous section and boost the dimension by one. Instead of categories, we need '2-categories'. Instead of groups, we need '2-groups'. Instead of the path groupoid, we need the 'path 2-groupoid'. And instead of functors, we need '2-functors'. When we understand all these things, the analogue of Theorem 1 will look strikingly similar to the original version:

Theorem For any Lie 2-group $\mathcal{G}$ and any smooth manifold $M$, there is a one-to-one correspondence between:

1. 2-connections on the trivial principal $\mathcal{G}$-2-bundle over $M$,

2. pairs consisting of a smooth $\mathfrak{g}$-valued 1-form $A$ and a smooth $\mathfrak{h}$-valued 2-form $B$ on $M$, such that

$$
\underline{t}(B)=d A+A \wedge A
$$

where we use $t: \mathfrak{h} \rightarrow \mathfrak{g}$, the differential of the map $t: H \rightarrow G$, to convert $B$ into a $\mathfrak{g}$-valued 2-form, and

3. smooth 2-functors

$$
\text { hol: } \mathcal{P}_{2}(M) \rightarrow \mathcal{G}
$$

where $\mathcal{P}_{2}(M)$ is the path 2-groupoid of $M$.

What does this say? In brief: there is a way to extract from a Lie 2-group $\mathcal{G}$ a pair of Lie groups $G$ and $H$. Suppose we have a 1-form $A$ taking values in the Lie algebra of $G$, and a 2-form $B$ valued in the Lie algebra of $H$. Suppose furthermore that these forms obey the equation above. Then we can use them to consistently define parallel transport, or 'holonomies', for paths and surfaces. They thus define a '2-connection'.

That is the idea. But to make it precise, we need 2-categories. 


\subsection{2-Categories}

Sets have elements. Categories have elements, usually called 'objects', but also morphisms between these. In an ' $n$-category', we go further and include 2-morphisms between morphisms, 3-morphisms between 2-morphisms,... and so on up to the $n$th level. We are beginning to see $n$-categories provide an algebraic language for $n$-dimensional structures in physics [13]. Higher gauge theory is just one place where this is happening.

Anyone learning $n$-categories needs to start with 2-categories [61]. A 2-category consists of:

- a collection of objects,

- for any pair of objects $x$ and $y$, a set of morphisms $f: x \rightarrow y$ :

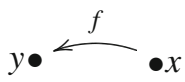

- for any pair of morphisms $f, g: x \rightarrow y$, a set of 2-morphisms $\alpha: f \Rightarrow g$ :

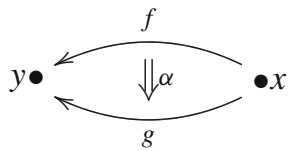

We call $f$ the source of $\alpha$ and $g$ the target of $\alpha$.

Morphisms can be composed just as in a category:

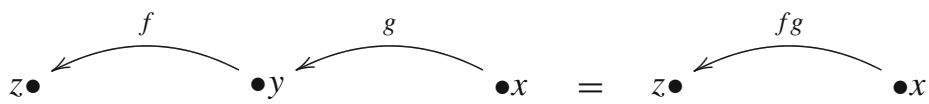

while 2-morphisms can be composed in two distinct ways, vertically:

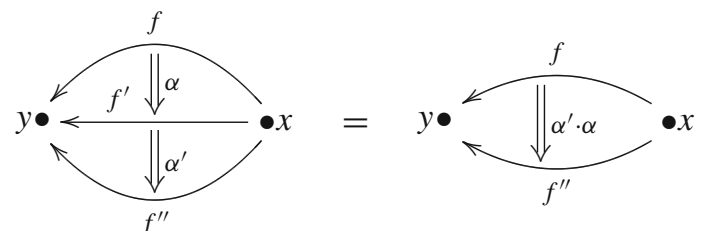

and horizontally:

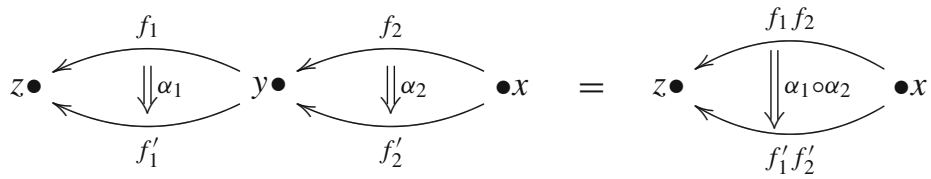


Finally, these laws must hold:

- Composition of morphisms is associative, and every object $x$ has a morphism

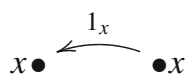

serving as an identity for composition, just as in an ordinary category.

- Vertical composition is associative, and every morphism $f$ has a 2-morphism

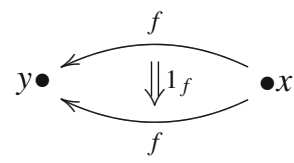

serving as an identity for vertical composition.

- Horizontal composition is associative, and the 2-morphism

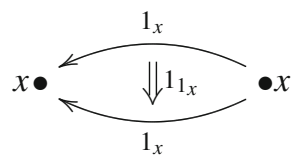

serves as an identity for horizontal composition.

- Vertical and horizontal composition of 2-morphisms obey the interchange law:

$$
\left(\alpha_{1}^{\prime} \cdot \alpha_{1}\right) \circ\left(\alpha_{2}^{\prime} \cdot \alpha_{2}\right)=\left(\alpha_{1}^{\prime} \circ \alpha_{2}^{\prime}\right) \cdot\left(\alpha_{1} \circ \alpha_{2}\right)
$$

so that diagrams of the form

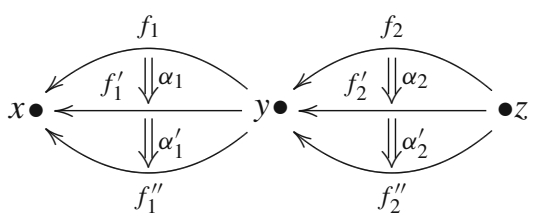

define unambiguous 2-morphisms.

The interchange law is the truly new thing here. A category is all about attaching 1-dimensional arrows end to end, and we need the associative law to do that unambiguously. In a 2-category, we visualize the 2-morphisms as little pieces of 2-dimensional surface:

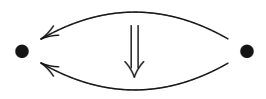


We can attach these together in two ways: vertically and horizontally. For the result to be unambiguous, we need not only associative laws but also the interchange law. In what follows we will see this law turning up all over the place.

\subsection{Path 2-groupoids}

Path groupoids play a big though often neglected role in physics: the path groupoid of a spacetime manifold describes all the possible motions of a point particle in that spacetime. The path 2-groupoid does the same thing for particles and strings.

First of all, a 2-groupoid is a 2-category where:

- Every morphism $f: x \rightarrow y$ has an inverse, $f^{-1}: y \rightarrow x$, such that:

$$
f^{-1} f=1_{x} \text { and } f f^{-1}=1_{y} .
$$

- Every 2-morphism $\alpha: f \Rightarrow g$ has a vertical inverse, $\alpha_{\text {vert }}^{-1}: g \Rightarrow f$, such that:

$$
\alpha_{\text {vert }}^{-1} \cdot \alpha=1_{f} \text { and } \alpha \cdot \alpha_{\text {vert }}^{-1}=1_{f} .
$$

It actually follows from this definition that every 2-morphism $\alpha: f \Rightarrow g$ also has a horizontal inverse, $\alpha_{\mathrm{hor}}^{-1}: f^{-1} \Rightarrow g^{-1}$, such that:

$$
\alpha_{\text {hor }}^{-1} \circ \alpha=1_{1_{x}} \text { and } \alpha \circ \alpha_{\text {hor }}^{-1}=1_{1_{x}} .
$$

So, a 2-groupoid has every kind of inverse your heart could desire.

An example of a 2-group is the 'path 2-groupoid' of a smooth manifold $M$. To define this, we can start with the path groupoid $\mathcal{P}_{1}(M)$ as defined in the previous section, and then throw in 2-morphisms. Just as the morphisms in $\mathcal{P}_{1}(M)$ were thin homotopy classes of lazy paths, these 2-morphisms will be thin homotopy classes of lazy surfaces.

What is a 'lazy surface'? First, recall that a homotopy between lazy paths $\gamma, \delta: x \rightarrow$ $y$ is a smooth map $\Sigma:[0,1]^{2} \rightarrow M$ with

$$
\begin{aligned}
& \Sigma(0, t)=\gamma_{0}(t) \\
& \Sigma(1, t)=\gamma_{1}(t)
\end{aligned}
$$

We say this homotopy is a lazy surface if

- $\Sigma(s, t)$ is independent of $s$ near $s=0$ and near $s=1$,

- $\Sigma(s, t)$ is constant near $t=0$ and constant near $t=1$.

Any homotopy $\Sigma$ yields a one-parameter family of paths $\gamma_{s}$ given by

$$
\gamma_{s}(t)=\Sigma(s, t)
$$

If $\Sigma$ is a lazy surface, each of these paths is lazy. Furthermore, the path $\gamma_{s}$ equals $\gamma_{0}$ when $s$ is sufficiently close to 0 , and it equals $\gamma_{1}$ when $s$ is sufficiently close to 1 . 
This allows us to compose lazy homotopies either vertically or horizontally and obtain new lazy homotopies!

However, vertical and horizontal composition will only obey the 2-groupoid axioms if we take 2-morphisms in the path 2-groupoid to be equivalence classes of lazy surfaces. We saw this kind of issue already when discussing the path groupoid, so we will allow ourselves to be a bit sketchy this time. The key idea is to define a concept of 'thin homotopy' between lazy surfaces $\Sigma$ and $\Xi$. For starters, this should be a smooth map $H:[0,1]^{3} \rightarrow M$ such that $H(0, s, t)=\Sigma(s, t)$ and $H(1, s, t)=\Xi(s, t)$. But we also want $H$ to be 'thin'. In other words, it should sweep out no volume: the rank of the differential $d H$ should be less than 3 at every point.

To make thin homotopies well-defined between thin homotopy classes of paths, some more technical conditions are also useful. For these, the reader can turn to Section 2.1 of Schreiber and Waldorf [87]. The upshot is that we obtain for any smooth manifold $M$ a path 2-groupoid $\mathcal{P}_{2}(M)$, in which:

- An object is a point of $M$.

- A morphism from $x$ to $y$ is a thin homotopy class of lazy paths from $x$ to $y$.

- A 2-morphism between equivalence classes of lazy paths $\gamma_{0}, \gamma_{1}: x \rightarrow y$ is a thin homotopy class of lazy surfaces $\Sigma: \gamma_{0} \Rightarrow \gamma_{1}$.

As we already did with the concept of 'lazy path', we will often use 'lazy surface' to mean a thin homotopy class of lazy surfaces. But now let us hasten on to another important class of 2-groupoids, the '2-groups'. Just as groups describe symmetries in gauge theory, these describe symmetries in higher gauge theory.

\subsection{2-Groups}

Just as a group was a groupoid with one object, we define a 2-group to be a 2-groupoid with one object. This definition is so elegant that it may be hard to understand at first! So, it will be useful to take a 2 -group $\mathcal{G}$ and chop it into four bite-sized pieces of data, giving a 'crossed module' $(G, H, t, \alpha)$. Indeed, 2-groups were originally introduced in the guise of crossed modules by the famous topologist Whitehead [94,95]. In 1950, with help from Mac Lane [64], he used crossed modules to generalize the fundamental group of a space to what we might now call the 'fundamental 2-group'. But only later did it become clear that a crossed module was another way of talking about a 2-groupoid with just one object! For more of this history, and much more on 2-groups, see [14].

Let us start by seeing what it means to say a 2-group is a 2-groupoid with one object. It means that a 2 -group $\mathcal{G}$ has:

- one object:

- morphisms: 
- and 2-morphisms:

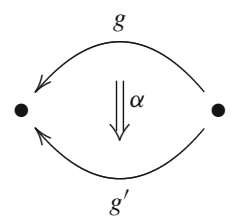

The morphisms form a group under composition:

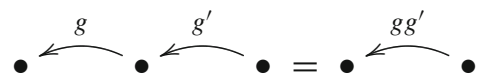

The 2-morphisms form a group under horizontal composition:

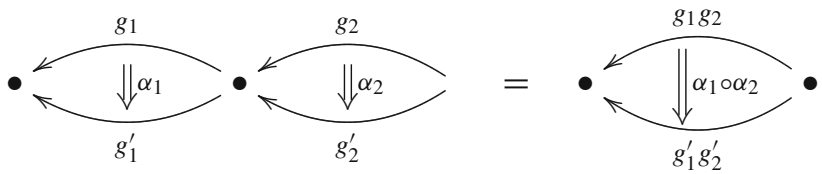

In addition, the 2-morphisms can be composed vertically:

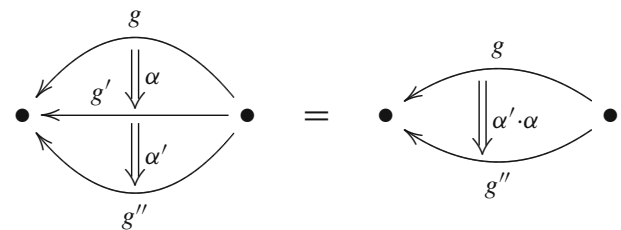

Vertical composition is also associative with identity and inverses. But the 2-morphisms do not form a group under this operation, because a given pair may not be composable: their source and target may not match up. Finally, vertical and horizontal composition are tied together by the interchange law, which says the two ways one can read this diagram are consistent.

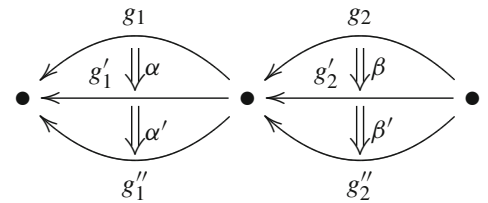

Now let us create a crossed module $(G, H, t, \alpha)$ from a 2-group $\mathcal{G}$. To do this, first note that the morphisms of the 2 -group form a group by themselves, with composition as the group operation. So: 
1. Let $G$ be the set of morphisms in $\mathcal{G}$, made into a group with composition as the group operation:

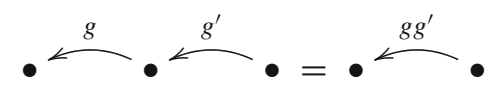

How about the 2-morphisms? These also form a group, with horizontal composition as the group operation. But it turns out to be efficient to focus on a subgroup of this:

- Let $H$ be the set of all 2-morphisms whose source is the identity:

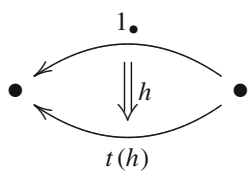

We make $H$ into a group with horizontal composition as the group operation:

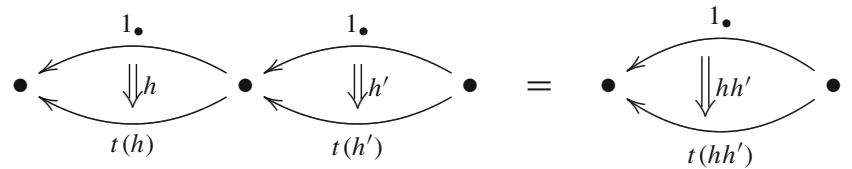

Above we use $h h^{\prime}$ as an abbreviation for the horizontal composite $h \circ h^{\prime}$ of two elements of $H$. We will use $h^{-1}$ to denote the horizontal inverse of an element of $H$. We use $t(h)$ to denote the target of an element $h \in H$. The definition of a 2-category implies that $t: H \rightarrow G$ is a group homomorphism:

$$
t\left(h h^{\prime}\right)=t(h) t\left(h^{\prime}\right) .
$$

This homomorphism is our third piece of data:

- A group homomorphism $t: H \rightarrow G$ sending each 2-morphism in $H$ to its target:

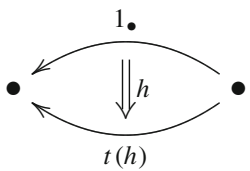

The fourth piece of data is the subtlest. There is a way to 'horizontally conjugate' any element $h \in H$ by an element $g \in G$, or more precisely by its identity 2 -morphism $1_{g}$ :

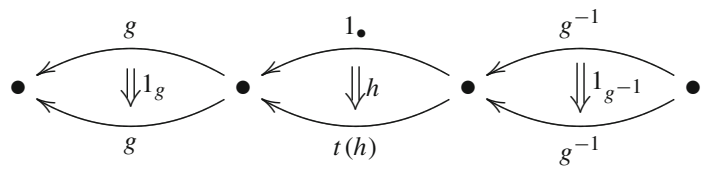


The result is a 2-morphism in $H$ which we call $\alpha(g)(h)$. In fact, $\alpha(g)$ is an automorphism of $H$, meaning a one-to-one and onto function with

$$
\alpha(g)\left(h h^{\prime}\right)=\alpha(g)(h) \alpha(g)\left(h^{\prime}\right) .
$$

Composing two automorphisms gives another automorphism, and this makes the automorphisms of $H$ into a group, say $\operatorname{Aut}(H)$. Even better, $\alpha$ gives a group homomorphism

$$
\alpha: G \rightarrow \operatorname{Aut}(H)
$$

Concretely, this means that in addition to the above equation, we have

$$
\alpha\left(g g^{\prime}\right)=\alpha(g) \alpha\left(g^{\prime}\right)
$$

Checking these two equations is a nice way to test your understanding of 2-categories. A group homomorphism $\alpha: G \rightarrow \operatorname{Aut}(H)$ is also called an action of the group $G$ on the group $H$. So, the fourth and final piece of data in our crossed module is:

- An action $\alpha$ of $G$ on $H$ given by:

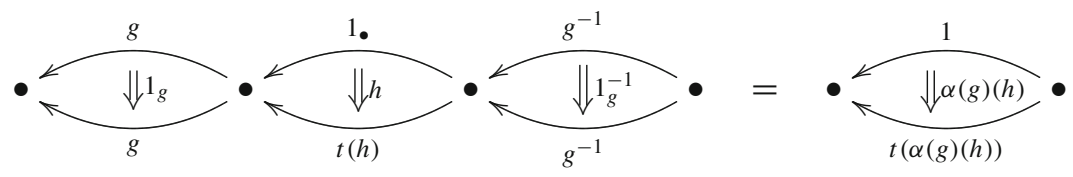

A crossed module $(G, H, t, \alpha)$ must also satisfy two more equations which follow from the definition of a 2-group. First, examining the above diagram, we see that $t$ is $G$-equivariant, by which we mean:

- $t(\alpha(g) h)=g(t(h)) g^{-1}$ for all $g \in G$ and $h \in H$.

Second, the Peiffer identity holds:

- $\alpha(t(h)) h^{\prime}=h h^{\prime} h^{-1}$ for all $h, h^{\prime} \in H$.

The Peiffer identity is the least obvious thing about a crossed module. It follows from the interchange law, and it is worth seeing how. First, we have:

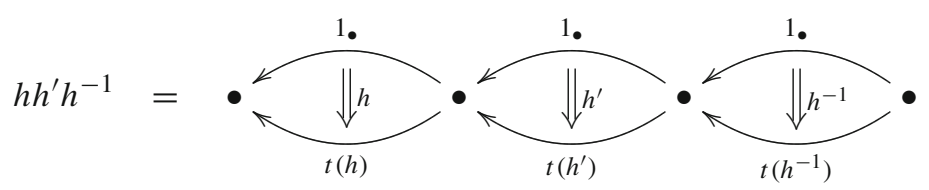


where-beware!-we are now using $h^{-1}$ to mean the horizontal inverse of $h$, since this is its inverse in the group $H$. We can pad out this equation by vertically composing with some identity morphisms:

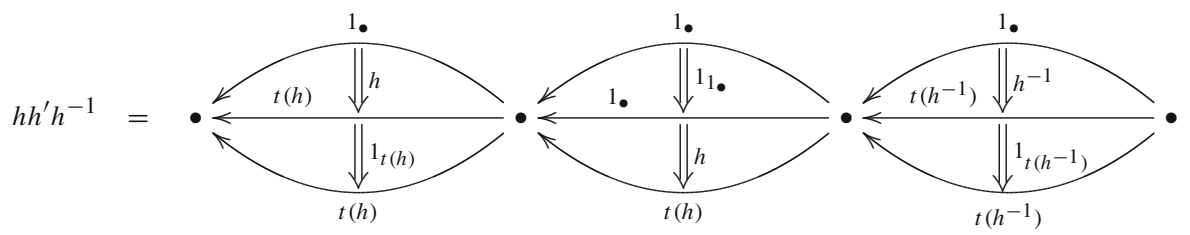

This diagram describes an unambiguous 2-morphism, thanks to the interchange law. So, we can do the horizontal compositions first and get:

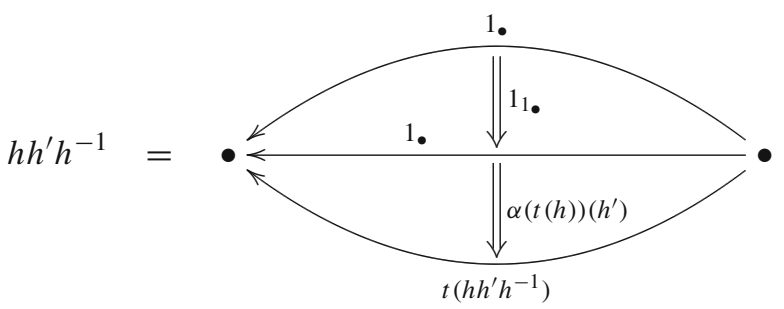

But vertically composing with an identity 2-morphism has no effect. So, we obtain the Peiffer identity:

$$
h h^{\prime} h^{-1}=\alpha(t(h))\left(h^{\prime}\right)
$$

All this leads us to define a crossed module $(G, H, t, \alpha)$ to consist of:

- a group $G$,

- a group $H$,

- a homomorphism $t: H \rightarrow G$, and

- an action $\alpha: G \rightarrow \operatorname{Aut}(H)$

such that:

- $t$ is $G$-equivariant:

$$
t(\alpha(g) h)=g(t(h)) g^{-1}
$$

for all $g \in G$ and $h \in H$, and

- the Peiffer identity holds for all $h, h^{\prime} \in H$ :

$$
\alpha(t(h)) h^{\prime}=h h^{\prime} h^{-1}
$$

In fact, we can recover a 2-group $\mathcal{G}$ from its crossed module $(G, H, t, \alpha)$, so crossed modules are just another way of thinking about 2-groups. The trick to seeing this is to notice that 2-morphisms in $\mathcal{G}$ are the same as pairs $(g, h) \in G \times H$. Such a pair gives this 2-morphism: 


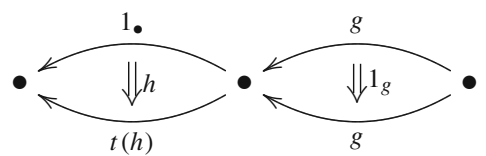

We leave it to the reader to check that every 2-morphism in $\mathcal{G}$ is of this form. Note that this 2-morphism goes from $g$ to $t(h) g$. So, when we construct a 2-group from a crossed module, we get a 2-morphism

$$
(g, h): g \rightarrow t(h) g
$$

from any pair $(g, h) \in G \times H$. Horizontal composition of 2-morphisms then makes $G \times H$ into a group, as follows:

$$
(g, h) \circ\left(g^{\prime}, h^{\prime}\right)=
$$
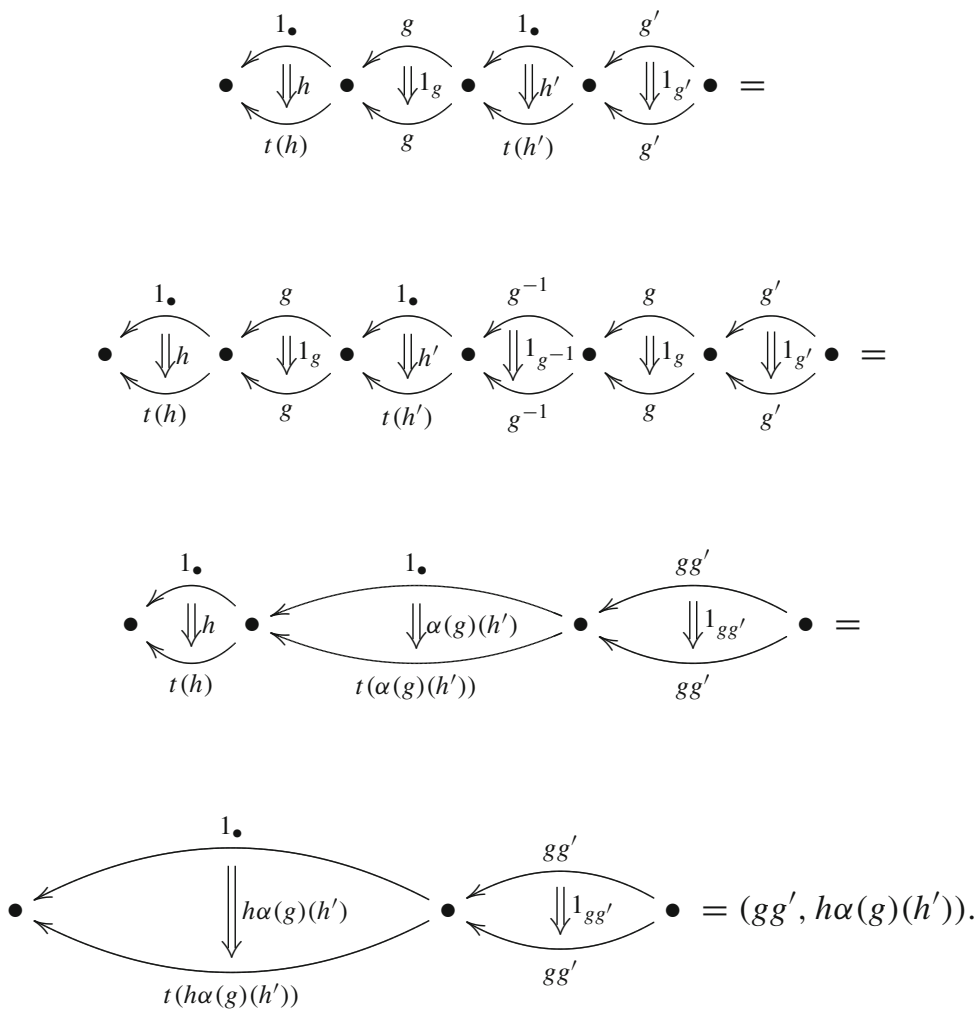

So, the group of 2-morphisms of $\mathcal{G}$ is the semidirect product $G \ltimes H$, defined using the action $\alpha$.

Following this line of thought, the reader can check the following: 
Theorem 2 Given a crossed module $(G, H, t, \alpha)$, there is a unique 2-group $\mathcal{G}$ where:

- the group of morphisms is $G$,

- a 2-morphism $\alpha: g \Rightarrow g^{\prime}$ is the same as a pair $(g, h) \in G \times H$ with $g^{\prime}=t(h) g$,

- the vertical composite of $(g, h)$ and $\left(g^{\prime}, h^{\prime}\right)$, when they are composable, is given by

$$
(g, h) \cdot\left(g^{\prime}, h^{\prime}\right)=\left(g^{\prime}, h h^{\prime}\right)
$$

- the horizontal composite of $(g, h)$ and $\left(g^{\prime}, h^{\prime}\right)$ is given by

$$
(g, h) \circ\left(g^{\prime}, h^{\prime}\right)=\left(g g^{\prime}, h \alpha(g)\left(h^{\prime}\right)\right) .
$$

Conversely, given a 2-group $\mathcal{G}$, there is a unique crossed module $(G, H, t, \alpha)$ where:

- $G$ is the group of morphisms of $\mathcal{G}$,

- $H$ is the group of 2-morphisms with source equal to 1.

- $t: H \rightarrow G$ assigns to each 2-morphism in $H$ its target,

- the action $\alpha$ of $G$ on $H$ is given by

$$
\alpha(g) h=1_{g} \circ h \circ 1_{g^{-1}}
$$

Indeed, these two processes set up an equivalence between 2-groups and crossed modules, as described more formally elsewhere [14,47]. It thus makes sense to define a Lie 2-group to be a 2-group for which the groups $G$ and $H$ in its crossed module are Lie groups, with the maps $t: H \rightarrow G$ and $\alpha: G \rightarrow \operatorname{Aut}(H)$ being smooth. It is worth emphasizing that in this context we use $\operatorname{Aut}(H)$ to mean the group of smooth automorphisms of $H$. This is a Lie group in its own right.

In Sect. 4 we will use Theorem 2 to construct many examples of Lie 2-groups. But first we should finish explaining 2-connections.

\subsection{2-Connections}

A 2-connection is a recipe for parallel transporting both 0-dimensional and 1-dimensional objects - say, particles and strings. Just as we can describe a connection on a trivial bundle using a Lie-algebra valued differential form, we can describe a 2-connection using a pair of differential forms. But there is a deeper way of understanding 2-connections. Just as a connection was revealed to be a smooth functor

$$
\text { hol: } \mathcal{P}_{1}(M) \rightarrow G
$$

for some Lie group $G$, a 2-connection will turn out to be a smooth 2-functor

$$
\text { hol: } \mathcal{P}_{2}(M) \rightarrow \mathcal{G}
$$

for some Lie 2-group $\mathcal{G}$. Of course, to make sense of this we need to define a '2-functor', and say what it means for such a thing to be smooth. 
The definition of 2-functor is utterly straightforward: it is a map between 2-categories that preserves everything in sight. So, given 2-categories $C$ and $D$, a 2-functor $F: C \rightarrow D$ consists of:

- a map $F$ sending objects in $C$ to objects in $D$,

- another map called $F$ sending morphisms in $C$ to morphisms in $D$,

- a third map called $F$ sending 2-morphisms in $C$ to 2-morphisms in $D$,

such that:

- given a morphism $f: x \rightarrow y$ in $C$, we have $F(f): F(x) \rightarrow F(y)$,

- $F$ preserves composition for morphisms, and identity morphisms:

$$
\begin{aligned}
F(f g) & =F(f) F(g) \\
F\left(1_{x}\right) & =1_{F(x)},
\end{aligned}
$$

- given a 2-morphism $\alpha: f \Rightarrow g$ in $C$, we have $F(\alpha): F(f) \Rightarrow F(g)$,

- $F$ preserves vertical and horizontal composition for 2-morphisms, and identity 2morphisms:

$$
\begin{aligned}
F(\alpha \cdot \beta) & =F(\alpha) \cdot F(\beta) \\
F(\alpha \circ \beta) & =F(\alpha) \circ F(\beta) \\
F\left(1_{f}\right) & =1_{F(f)} .
\end{aligned}
$$

There is a general theory of smooth 2-groupoids and smooth 2-functors [17,88,89]. But here we prefer to take a more elementary approach. We already know that for any Lie 2-group $\mathcal{G}$, the morphisms form a Lie group. In the next section we say that the 2morphisms also form a Lie group, with horizontal composition as the group operation. Given this, we can say that for any smooth manifold $M$, a 2-functor

$$
\text { hol: } \mathcal{P}_{2}(M) \rightarrow \mathcal{G}
$$

is smooth if:

- For any smoothly parametrized family of lazy paths $\gamma_{s}\left(s \in[0,1]^{n}\right)$ the morphism $\operatorname{hol}\left(\gamma_{s}\right)$ depends smoothly on $s$, and

- For any smoothly parametrized family of lazy surfaces $\Sigma_{s}\left(s \in[0,1]^{n}\right)$ the morphism hol $\left(\Sigma_{s}\right)$ depends smoothly on $s$.

With these definitions in hand, we are finally ready to understand the basic result about 2-connections. It is completely analogous to Theorem 1:

Theorem 3 For any Lie 2-group $\mathcal{G}$ and any smooth manifold $M$, there is a one-to-one correspondence between:

1. 2-connections on the trivial principal $\mathcal{G}$-2-bundle over $M$,

2. pairs consisting of a smooth $\mathfrak{g}$-valued 1-form $A$ and a smooth $\mathfrak{h}$-valued 2-form $B$ on $M$, such that

$$
\underline{t}(B)=d A+A \wedge A
$$


where we use $\underline{t}: \mathfrak{h} \rightarrow \mathfrak{g}$, the differential of the map $t: H \rightarrow G$, to convert $B$ into a $\mathfrak{g}$-valued 2-form, and

3. smooth 2-functors

$$
\text { hol: } \mathcal{P}_{2}(M) \rightarrow \mathcal{G}
$$

where $\mathcal{P}_{2}(M)$ is the path 2-groupoid of $M$.

This result was announced by Baez and Schreiber [17], and a proof can be found in the work of Schreiber and Waldorf $[88,89]$. This work was deeply inspired by the ideas of Breen and Messing [28,29], who considered a special class of 2-groups, and omitted the equation $\underline{t}(B)=d A+A \wedge A$, since their sort of connection did not assign holonomies to surfaces. One should also compare the closely related work of Mackaay, Martins, and Picken [65,67,68], and the work of Pfeiffer and Girelli [76,56].

In the above theorem, the first item mentions '2-connections' and '2-bundles'concepts that we have not defined. But since we are only talking about 2-connections on trivial 2-bundles, we do not need these general concepts yet. For now, we can take the third item as the definition of the first. Then the content of the theorem lies in the differential form description of smooth 2-functors hol: $\mathcal{P}_{2}(M) \rightarrow \mathcal{G}$. This is what we need to understand.

A 2-functor of this sort must assign holonomies both to paths and surfaces. As you might expect, the 1 -form $A$ is primarily responsible for defining holonomies along paths, while the 2-form $B$ is responsible for defining holonomies for surfaces. But this is a bit of an oversimplification. When computing the holonomy of a surface, we need to use $A$ as well as $B$ !

Another surprising thing is that $A$ and $B$ need to be related by an equation for the holonomy to be a 2-functor. If we ponder how the holonomy of a surface is actually computed, we can see why this is so. We shall not be at all rigorous here. We just want to give a rough intuitive idea of how to compute a holonomy for a surface, and where the equation $\underline{t}(B)=d A+A \wedge A$ comes from. Of course

$$
d A+A \wedge A=F
$$

is just the curvature of the connection $A$. This is a big clue.

Suppose we are trying to compute the holonomy for a surface starting from a $\mathfrak{g}$ valued 1-form $A$ and an $\mathfrak{h}$-valued 2-form $B$. Then following the ideas of calculus, we can try to chop the surface into many small pieces, compute a holonomy for each one, and multiply these together somehow. It is easy to chop a surface into small squares. Unfortunately, the definition of 2-category doesn't seem to know anything about squares! But this is not a serious problem. For example, we can interpret this square:

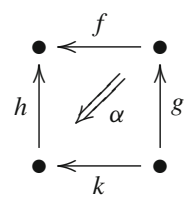


as a 2-morphism $\alpha: f g \Rightarrow h k$. We can then compose a bunch of such 2-morphisms:

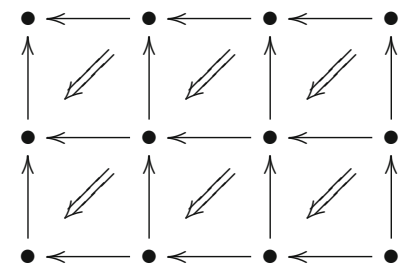

with the help of a trick called 'whiskering'.

Whiskering is a way to compose a 1-morphism and a 2-morphism. Suppose we want to compose a 2-morphism $\alpha$ and a morphism $f$ that sticks out like a whisker on the left:

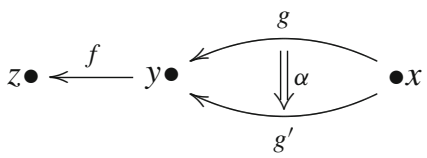

We can do this by taking the horizontal composite $1_{f} \circ \alpha$ :

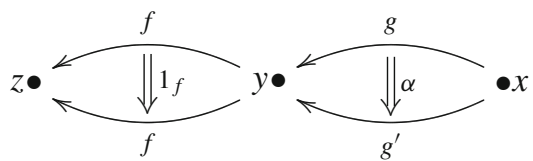

We call the result $f \circ \alpha$, or $\alpha$ left whiskered by $f$. Similarly, if we have a whisker sticking out on the right:

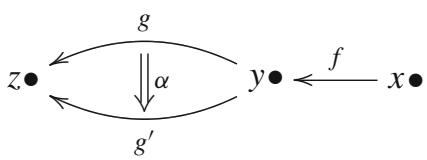

we can take the horizontal composite $\alpha \circ 1_{f}$ :

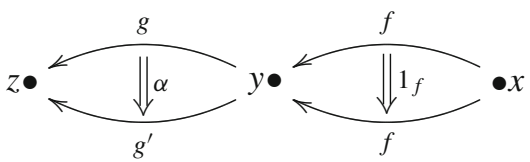

and call the result $\alpha \circ f$, or $\alpha$ right whiskered by $f$.

With the help of whiskering, we can compose 2-morphisms shaped like arbitrary polygons. For example, suppose we want to horizontally compose two squares: 


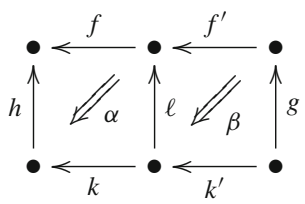

To do this, we can left whisker $\beta$ by $f$, obtaining this 2-morphism:

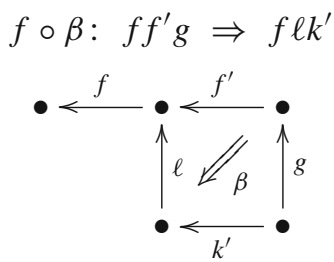

Then we can right whisker $\alpha$ by $k^{\prime}$, obtaining

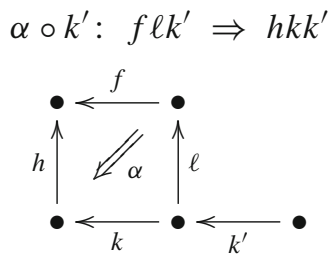

Then we can vertically compose these to get the desired 2-morphism:

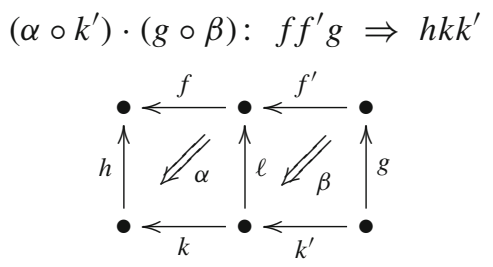

The same sort of trick lets us vertically compose squares. By iterating these procedures we can define more complicated composites, like this:

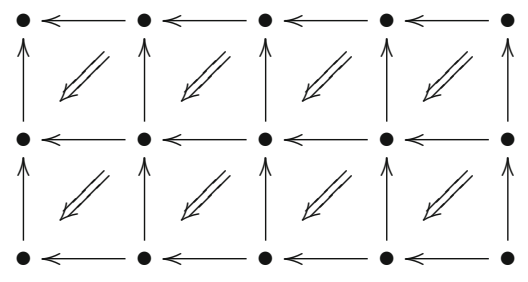


Of course, one may wonder if these more complicated composites are unambiguously defined! Luckily they are, thanks to associativity and the interchange law. This is a nontrivial result, called the 'pasting theorem' [60,77].

By this method, we can reduce the task of computing hol $(\Sigma)$ for a large surface $\Sigma$ to the task of computing it for lots of small squares. Ultimately, of course, we should take a limit as the squares become smaller and smaller. But for our nonrigorous discussion, it is enough to consider a very small square like this:

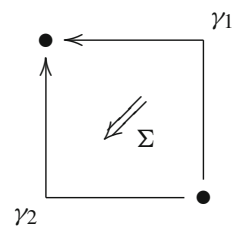

We can think of this square as a 2-morphism

$$
\Sigma: \gamma_{1} \Rightarrow \gamma_{2}
$$

where $\gamma_{1}$ is the path that goes up and then across, while $\gamma_{2}$ goes across and then up. We wish to compute

$$
\operatorname{hol}(\Sigma): \operatorname{hol}\left(\gamma_{1}\right) \Rightarrow \operatorname{hol}\left(\gamma_{2}\right)
$$

On the one hand, $\operatorname{hol}(\Sigma)$ involve the 2-form $B$. On the other hand, its source and target depend only on the 1 -form $A$ :

$$
\operatorname{hol}\left(\gamma_{1}\right)=\mathcal{P} \exp \left(\int_{\gamma_{i}} A\right), \quad \operatorname{hol}\left(\gamma_{2}\right)=\mathcal{P} \exp \left(\int_{\gamma_{2}} A\right) .
$$

So, hol $(\Sigma)$ cannot have the right source and target unless $A$ and $B$ are related by an equation!

Let us try to guess this equation. Recall from Theorem 2 that a 2-morphism $\alpha: g_{1} \Rightarrow$ $g_{2}$ in $\mathcal{G}$ is determined by an element $h \in H$ with $g_{2}=t(h) g_{1}$. Using this, we may think of $\operatorname{hol}(\Sigma): \operatorname{hol}\left(\gamma_{1}\right) \rightarrow \operatorname{hol}\left(\gamma_{2}\right)$ as determined by an element $h \in H$ with

$$
\mathcal{P} \exp \left(\int_{\gamma_{2}} A\right)=t(h) \mathcal{P} \exp \left(\int_{\gamma_{1}} A\right)
$$

or in other words

$$
t(h)=\mathcal{P} \exp \left(\int_{\partial \Sigma} A\right)
$$


where the loop $\partial \Sigma=\gamma_{2} \gamma_{1}^{-1}$ goes around the square $\Sigma$. For a very small square, we can approximately compute the right hand side using Stokes' theorem:

$$
\mathcal{P} \exp \left(\int_{\partial \Sigma} A\right) \approx \exp \left(\int_{\Sigma} F\right) .
$$

On the other hand, there is an obvious guess for the approximate value of $h$, which is supposed to be built using the 2-form $B$ :

$$
h \approx \exp \left(\int_{\Sigma} B\right)
$$

For this guess to yield (1), at least to first order in the size of our square, we need

$$
t\left(\exp \left(\int_{\Sigma} B\right)\right) \approx \exp \left(\int_{\Sigma} F\right) .
$$

But this will be true if

$$
\underline{t}(B)=F \text {. }
$$

And this is the equation that relates $A$ and $B$ !

What have we learned here? First, for any surface $\Sigma: \gamma_{1} \Rightarrow \gamma_{2}$, the holonomy $\operatorname{hol}(\Sigma)$ is determined by an element $h \in H$ with

$$
\mathcal{P} \exp \left(\int_{\gamma_{2}} A\right)=t(h) \mathcal{P} \exp \left(\int_{\gamma_{1}} A\right)
$$

In the limit where $\Sigma$ is very small, this element $h$ depends only on $B$ :

$$
h \approx \exp \left(\int_{\Sigma} B\right) .
$$

But for a finite-sized surface, this formula is no good, since it involves adding up $B$ at different points, which is not a smart thing to do. For a finite-sized surface, $h$ depends on $A$ as well as $B$, since we can approximately compute $h$ by chopping this surface into small squares, whiskering them with paths, and composing them-and the holonomies along these paths are computed using $A$.

To get the exact holonomy over a finite-sized surface by this method, we need to take a limit where we subdivide the surface into ever smaller squares. This is the Lie 2-group analogue of a Riemann sum. But for actual calculations, this process is not 
very convenient. More practical formulas for computing holonomies over surfaces can be found in the work of Schreiber and Waldorf [88,89], Martins and Picken $[67,68]$.

\section{Examples and applications}

Now let us give some examples of Lie 2-groups, and see what higher gauge theory can do with these examples. We will build these examples using crossed modules. Throughout what follows, $\mathcal{G}$ is a Lie 2-group whose corresponding crossed module is $(G, H, t, \alpha)$.

\subsection{Shifted abelian groups}

Any group $G$ automatically gives a 2-group where $H$ is trivial. Then higher gauge theory reduces to ordinary gauge theory. But to see what is new about higher gauge theory, let us instead suppose that $G$ is the trivial group. Then $t$ and $\alpha$ are forced to be trivial, and $t$ is automatically $G$-equivariant. On the other hand, the Peiffer identity

$$
\alpha(t(h)) h^{\prime}=h h^{\prime} h^{-1}
$$

is not automatic: it holds if and only if $H$ is abelian!

There is also a nice picture proof that $H$ must be abelian when $G$ is trivial. We simply move two elements of $H$ around each other using the interchange law:

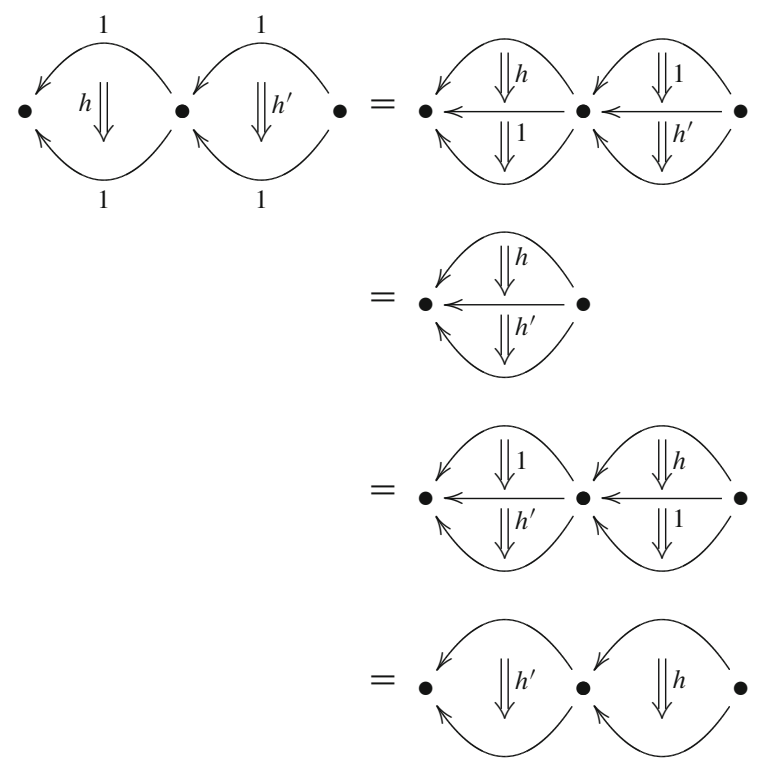

As a side-benefit, we see that horizontal and vertical composition must be equal when $G$ is trivial. This proof is called the 'Eckmann-Hilton argument', since Eckmann and Hilton used it to show that the second homotopy group of a space is abelian [43]. 
So, we can build a 2-group where:

- $G$ is the trivial group,

- $H$ is any abelian Lie group,

- $\alpha$ is trivial, and

- $t$ is trivial.

This is called the shifted version of $H$, and denoted $\mathrm{b} H$.

In applications to physics, we often see $H=\mathrm{U}(1)$. A principal bU(1)-2-bundle is usually called a U(1) gerbe, and a 2-connection on such a thing is usually just called a connection. By Theorem 3, a connection on a trivial U(1) gerbe is just an ordinary real-valued 2-form $B$. Its holonomy is given by:

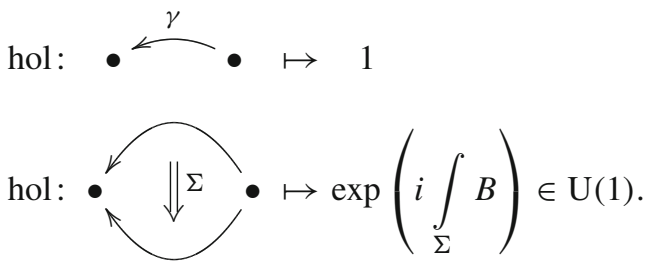

The book by Brylinski [30] gives a rather extensive introduction to U(1) gerbes and their applications. Murray's theory of 'bundle gerbes' gives a different viewpoint $[73,74,90]$. Here let us discuss two places where U(1) gerbes show up in physics. One is 'multisymplectic geometry'; the other is '2-form electromagnetism'. The two are closely related.

First, let us remember how 1-forms show up in symplectic geometry and electromagnetism. Suppose we have a point particle moving in some manifold $M$. At any time its position is a point $q \in M$ and its momentum is a cotangent vector $p \in T_{q}^{*} M$. As time passes, its position and momentum trace out a curve

$$
\gamma:[0,1] \rightarrow T^{*} M
$$

The action of this path is given by

$$
S(\gamma)=\int_{\gamma}\left(p_{i} \dot{q}^{i}-H(q, p)\right) d t
$$

where $H: T^{*} M \rightarrow \mathbb{R}$ is the Hamiltonian. But now suppose the Hamiltonian is zero! Then there is still a nontrivial action, due to the first term. We can rewrite it as follows:

$$
S(\gamma)=\int_{\gamma} \alpha
$$

where the 1-form

$$
\alpha=p_{i} d q^{i}
$$


is a canonical structure on the cotangent bundle. We can think of $\alpha$ as connection on a trivial U(1)-bundle over $T^{*} M$. Physically, this connection describes how a quantum particle changes phase even when the Hamiltonian is zero! The change in phase is computed by exponentiating the action. So, we have:

$$
\operatorname{hol}(\gamma)=\exp \left(i \int_{\gamma} \alpha\right) .
$$

Next, suppose we carry our particle around a small loop $\gamma$ which bounds a disk $D$. Then Stokes' theorem gives

$$
S(\gamma)=\int_{\gamma} \alpha=\int_{D} d \alpha
$$

Here the 2-form

$$
\omega=d \alpha=d p_{i} \wedge d q^{i}
$$

is the curvature of the connection $\alpha$. It makes $T^{*} M$ into a symplectic manifold, that is, a manifold with a closed 2-form $\omega$ satisfying the nondegeneracy condition

$$
\forall v \omega(u, v)=0 \Longrightarrow u=0
$$

The subject of symplectic geometry is vast and deep, but sometimes this simple point is neglected: the symplectic structure describes the change in phase of a quantum particle as we move it around a loop:

$$
\operatorname{hol}(\gamma)=\exp \left(i \int_{D} \omega\right)
$$

Perhaps this justifies calling a symplectic manifold a 'phase space', though historically this seems to be just a coincidence.

It may seem strange to talk about a quantum particle tracing out a loop in phase space, since in quantum mechanics we cannot simultaneously know a particle's position and momentum. However, there is a long line of work, beginning with Feynman, which computes time evolution by an integral over paths in phase space [41]. This idea is also implicit in geometric quantization, where the first step is to equip the phase space with a principal U(1)-bundle having a connection whose curvature is the symplectic structure. (Our discussion so far is limited to trivial bundles, but everything we say generalizes to the nontrivial case.)

Next, consider a charged particle in an electromagnetic field. Suppose that we can describe the electromagnetic field using a vector potential $A$ which is a connection on trivial $\mathrm{U}(1)$ bundles over $M$. Then we can pull $A$ back via the projection 
$\pi: T^{*} M \rightarrow M$, obtaining a 2 -form $\pi^{*} A$ on phase space. In the absence of any other Hamiltonian, the particle's action as we move it along a path $\gamma$ in phase space will be

$$
S(\gamma)=\int_{\gamma} \alpha+e \pi^{*} A
$$

if the particle has charge $e$. In short, the electromagnetic field changes the connection on phase space from $\alpha$ to $\alpha+e \pi^{*} A$. Similarly, when the path $\gamma$ is a loop bounding a disk $D$, we have

$$
S(\gamma)=\int_{D} \omega+e \pi^{*} F
$$

where $F=d A$ is the electromagnetic field strength. So, electromagnetism also changes the symplectic structure on phase space from $\omega$ to $\omega+e \pi^{*} F$. For more on this, see Guillemin and Sternberg [58], who also treat the case of nonabelian gauge fields.

All of this has an analog where particles are replaced by strings. It has been known for some time that just as the electromagnetic vector potential naturally couples to point particles, there is a 2 -form $B$ called the Kalb-Ramond field which naturally couples to strings. The action for this coupling is obtained simply by integrating $B$ over the string worldsheet. In 1986, Gawedski [52] showed that the $B$ field should be seen as a connection on a U(1) gerbe. Later Freed and Witten [48] showed this viewpoint was crucial for understanding anomaly cancellation. However, these authors did not actually use the word 'gerbe'. The role of gerbes was later made explicit by Carey et al. [34], and even more so by Gawedski and Reis [53].

In short, electromagnetism has a 'higher version'. What about symplectic geometry? This also has a higher version, which dates back to 1935 work by DeDonder [40] and Weyl [93]. The idea here is that an $n$-dimensional classical field theory has a kind of finite-dimensional phase space equipped with a closed $(n+2)$-form $\omega$ which is nondegenerate in the following sense:

$$
\forall v_{1}, \ldots, v_{n+1} \omega\left(u, v_{1}, \ldots, v_{n}\right)=0 \quad \Longrightarrow \quad u=0 \text {. }
$$

Such a $n$-form is called a multisymplectic structure, or more specifically, an $n$-plectic structure. For a nice introduction to multisymplectic geometry, see the paper by Gotay, Isenberg et al. [55].

The link between multisymplectic geometry and higher electromagnetism was made in a paper by Baez etal. [11]. Everything is closely analogous to the story for point particles. For a classical bosonic string propagating on Minkowski spacetime of any dimension, say $M$, there is a finite-dimensional manifold $X$ which serves as a kind of 'phase space' for the string. There is a projection $\pi: X \rightarrow M$, and there is a god-given way to take any map from the string's worldsheet to $M$ and lift it to an embedding of the worldsheet in $X$. So, let us write $\Sigma$ for the string worldsheet considered as a surface in $X$. 
The phase space $X$ is equipped with a 2-plectic structure: that is, a closed nondegenerate 3-form, say $\omega$. But in fact, $\omega=d \alpha$ for some 2-form $\alpha$. Even when the string's Hamiltonian is zero, there is a term in the action of the string coming from the integral of $\alpha$ :

$$
S(\Sigma)=\int_{\Sigma} \alpha .
$$

We may also consider a charged string coupled to a Kalb-Ramond field. This begins life as a 2 -form $B$ on $M$, but we may pull it back to a 2 -form $\pi^{*} B$ on $X$, and then

$$
S(\Sigma)=\int_{\Sigma} \alpha+e \pi^{*} B .
$$

In particular, suppose $\Sigma$ is a 2-sphere bounding a 3-ball $D$ in $X$. Then by Stokes' theorem we have

$$
S(\Sigma)=\int_{D} \omega+e \pi^{*} Z
$$

where the 3-form

$$
Z=d B
$$

is the Kalb-Ramond analog of the electromagnetic field strength, and $e$ is the string's charge. (The Kalb-Ramond field strength is usually called ' $H$ ' in the physics literature, but that conflicts with our usage of $H$ to mean a Lie group, so we shall call it ' $Z$ '.)

In summary: the Kalb-Ramond field modifies the 2-plectic structure on the phase space of the string. The reader will note that we have coyly refused to describe the phase space $X$ or its 2-form $\alpha$. For this, see the paper by Baez et al. [11]. In this paper, we explain how the usual dynamics of a classical bosonic string coupled to a KalbRamond field can be described using multisymplectic geometry. We also explain how to generalize Poisson brackets from symplectic geometry to multisymplectic geometry. Just as Poisson brackets in symplectic geometry make the functions on phase space into a Lie algebra, Poisson brackets in multisymplectic geometry give rise to a 'Lie 2-algebra'. Lie 2-algebras are also important in higher gauge theory in the same way that Lie algebras are important for gauge theory. Indeed, the 'string 2-group' described in Sect. 4.6 was constructed only after its Lie 2-algebra was found [8]. Later, this Lie 2-algebra was seen to arise naturally from multisymplectic geometry [16].

\subsection{The Poincaré 2-group}

Suppose we have a representation $\alpha$ of a Lie group $G$ on a finite-dimensional vector space $H$. We can regard $H$ as an abelian Lie group with addition as the group 
operation. This lets us regard $\alpha$ as an action of $G$ on this abelian Lie group. So, we can build a 2-group $\mathcal{G}$ where:

- $G$ is any Lie group,

- $H$ is any vector space,

- $\alpha$ is the representation of $G$ on $H$, and

- $t$ is trivial.

In particular, note that the Peiffer identity holds. In this way, we see that any group representation gives a crossed module-so group representations are secretly 2-groups!

For example, if we let $G$ be the Lorentz group and let $\alpha$ be its obvious representation on $\mathbb{R}^{4}$ :

$$
\begin{aligned}
G & =\operatorname{SO}(3,1) \\
H & =\mathbb{R}^{4}
\end{aligned}
$$

we obtain the so-called Poincaré 2-group, which has the Lorentz group as its group of morphisms, and the Poincaré group as its group of 2-morphisms [14].

What is the Poincaré 2-group good for? It is not clear, but there are some clues. Just as we can study representations of groups on vector spaces, we can study representations of 2-groups on '2-vector spaces' [7,25,39,44]. The representations of a group are the objects of a category, and this sort of category can be used to build 'spin foam models' of background-free quantum field theories [5,6]. This endeavor has been most successful with $3 \mathrm{~d}$ quantum gravity [49-51], but everyone working on this subject dreams of doing something similar for $4 \mathrm{~d}$ quantum gravity [80]. Going from groups to 2-groups boosts the dimension of everything: the representations of a 2-group are the objects of a 2-category, and Crane and Sheppeard outlined a program for building a 4-dimensional spin foam model starting from the 2-category of representations of the Poincaré 2-group [37].

Crane and Sheppeard hoped their model would be related to quantum gravityin 4 spacetime dimensions. This has not come to pass, at least not yet-but this spin foam model does have interesting connections to $4 \mathrm{~d}$ physics. The spin foam model of $3 \mathrm{~d}$ quantum gravity automatically includes point particles, and Baratin and Freidel have shown that it reduces to the usual theory of Feynman diagrams in 3d Minkowski spacetime in the limit where the gravitational constant $G_{\text {Newton }}$ goes to zero [22]. This line of thought led Baratin and Freidel to construct a spin foam model that is equivalent to the usual theory of Feynman diagrams in $4 \mathrm{~d}$ Minkowski spacetime [23]. At first the mathematics underlying this model was a bit mysterious - but it now seems clear that this model is based on the representation theory of the Poincare 2-group! For a preliminary report on this fascinating research, see the paper by Baratin and Wise [24].

In short, it appears that the 2-category of representations of the Poincare 2-group gives a spin foam description of quantum field theory on 4d Minkowski spacetime. Unfortunately, while spin foam models in 3 dimensions can be obtained by quantizing gauge theories, we do not see how to obtain this $4 \mathrm{~d}$ spin foam model by quantizing a higher gauge theory. Indeed, we know of no classical field theory in 4 dimensions whose solutions are 2-connections on a principal $\mathcal{G}$-2-bundle where $\mathcal{G}$ is the Poincaré 2-group. 
However, if we replace the Poincaré 2-group by a closely related 2-group, this puzzle does have a nice solution. Namely, if we take

$$
\begin{aligned}
G & =\operatorname{SO}(3,1) \\
H & =\mathfrak{s o}(3,1)
\end{aligned}
$$

and take $\alpha$ to be the adjoint representation, we obtain the 'tangent 2-group' of the Lorentz group. As we shall see, 2-connections for this 2-group arise naturally as solutions of a $4 \mathrm{~d}$ field theory called 'topological gravity'.

\subsection{Tangent 2-groups}

We have seen that any group representation gives a 2-group. But any Lie group $G$ has a representation on its own Lie algebra: the adjoint representation. This lets us build a 2-group from the crossed module where:

- $G$ is any Lie group,

- $H$ is $\mathfrak{g}$ regarded as a vector space and thus an abelian Lie group,

- $\alpha$ is the adjoint representation, and

- $t$ is trivial.

We call this the tangent 2-group $\mathcal{T} G$ of the Lie group $G$. Why? We have already seen that for any Lie 2-group, the group of all 2-morphisms is the semidirect product $G \ltimes H$. In the case at hand, this semidirect product is just $G \ltimes \mathfrak{g}$, with $G$ acting on $\mathfrak{g}$ via the adjoint representation. But as a manifold, this semidirect product is nothing other than the tangent bundle $T G$ of the Lie group $G$. So, the tangent bundle $T G$ becomes a group, and this is the group of 2-morphisms of $\mathcal{T} G$.

By Theorem 3, a 2-connection on a trivial $\mathcal{T} G$-2-bundle consists of a $\mathfrak{g}$-valued 1-form $A$ and a $\mathfrak{g}$-valued 2-form $B$ such that the curvature $F=d A+A \wedge A$ satisfies

$$
F=0
$$

since $\underline{t}(B)=0$ in this case. Where can we find such 2 -connections? We can find them as solutions of a field theory called 4-dimensional $B F$ theory!

$B F$ theory is a classical field theory that works in any dimension. So, take an $n$-dimensional oriented manifold $M$ as our spacetime. The fields in $B F$ theory are a connection $A$ on the trivial principal $G$-bundle over $M$, together with a $\mathfrak{g}$-valued $(n-2)$-form $B$. The action is given by

$$
S(A, B)=\int_{M} \operatorname{tr}(B \wedge F) .
$$

Setting the variation of this action equal to zero, we obtain the following field equations:

$$
d B+[A, B]=0, \quad F=0 .
$$


In dimension $4, B$ is a $\mathfrak{g}$-valued 2-form-and thanks to the second equation, $A$ and $B$ fit together to define a 2 -connection on the trivial $\mathcal{T} G$-2-bundle over $M$.

It may seem dull to study a gauge theory where the equations of motion imply the connection is flat. But there is still room for some fun. We see this already in 3-dimensional $B F$ theory, where $B$ is a $\mathfrak{g}$-valued 1-form rather than a 2-form. This lets us package $A$ and $B$ into a connection on the trivial $T G$-bundle over $M$. The field equations

$$
d B+[A, B]=0, \quad F=0
$$

then say precisely that this connection is flat.

When the group $G$ is the Lorentz group $\mathrm{SO}(2,1), T G$ is the corresponding Poincare group. With this choice of $G, 3 \mathrm{~d} B F$ theory is a version of $3 \mathrm{~d}$ general relativity. In 3 dimensions, unlike the more physical $4 \mathrm{~d}$ case, the equations of general relativity say that spacetime is flat in the absence of matter. And at first glance, $3 \mathrm{~d} B F$ theory only describes general relativity without matter. After all, its solutions are flat connections.

Nonetheless, we can consider $3 \mathrm{~d} B F$ theory on a manifold from which the worldline of a point particle has been removed. In the Bohm-Aharonov effect, if we carry a charged object around a solenoid, we obtain a nontrivial holonomy even though the U(1) connection $A$ is flat outside the solenoid. Similarly, in $3 \mathrm{~d} B F$ theory, the connection $(A, B)$ will be flat away from the particle's worldline, but it can have a nontrivial holonomy around a loop $\gamma$ that encircles the worldline:

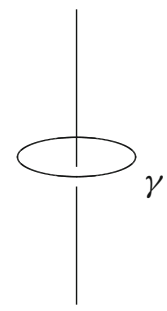

This holonomy says what happens when we parallel transport an object around our point particle. The holonomy is an element of Poincaré group. Its conjugacy class describes the mass and spin of our particle. So, massive spinning point particles are lurking in the formalism of $3 \mathrm{~d} B F$ theory!

Even better, this theory predicts an upper bound on the particle's mass, roughly the Planck mass. This is true even classically. This may seem strange, but unlike in 4 dimensions, where we need $c, G_{\text {Newton }}$ and $\hbar$ to build a quantity with dimensions of length, in 3-dimensional spacetime we can do this using only $c$ and $G_{\text {Newton }}$. So, ironically, the 'Planck mass' does not depend on Planck's constant.

Furthermore, in this theory, particles have 'exotic statistics', meaning that the interchange of identical particles is governed by the braid group instead of the symmetric group. Particles with exotic statistics are also known as 'anyons'. In the simplest examples, the anyons in $3 \mathrm{~d}$ gravity reduce to bosons or fermions in the $G_{\text {Newton }} \rightarrow 0$ limit. 
There is thus a wealth of interesting phenomena to be studied in $3 \mathrm{~d} B F$ theory. See the paper by Baez et al. [10] for a quick overview, and the work of Freidel, Louapre and Baratin for a deep treatment of the details [22,49-51].

The case of $4 \mathrm{~d} B F$ theory is just as interesting, and not as fully explored. In this case the field equations imply that $A$ and $B$ define a 2-connection on the trivial $\mathcal{T}$ $G$-2-bundle over $M$. But in fact they say more: they say precisely that this 2 -connection is flat. By this we mean two things. First, the holonomy hol $(\gamma)$ along a path $\gamma$ does not change when we change this path by a homotopy. Second, the holonomy hol $(\Sigma)$ along a surface $\Sigma$ does not change when we change this surface by a homotopy. The first fact here is equivalent to the equation $F=0$. The second is equivalent to the equation $d B+[A, B]=0$.

When the group $G$ is the Lorentz group $\mathrm{SO}(3,1), 4 \mathrm{~d} B F$ theory is sometimes called 'topological gravity'. We can think of it as a simplified version of general relativity that acts more like gravity in 3 dimensions. In particular, we can copy what we did in 3 dimensions, and consider $4 \mathrm{~d} B F$ theory on a manifold from which the worldlines of particles and the worldsheets of strings have been removed. Some of what we will do here works for more general groups $G$, but let us take $G=\operatorname{SO}(3,1)$ just to be specific.

First consider strings. Take a 2-dimensional manifold $X$ embedded in a 4-dimensional manifold $M$, and think of $X$ as the worldsheet of a string. Suppose we can find a small loop $\gamma$ that encircles $\Sigma$ in such a way that $\gamma$ is contractible in $M$ but not in $M-X$. If we do $4 \mathrm{~d} B F$ theory on the spacetime $M-X$, the holonomy

$$
\operatorname{hol}(\gamma) \in \operatorname{SO}(3,1)
$$

will not change when we apply a homotopy to $\gamma$. This holonomy describes the 'mass density' of our string [10,21].

Next, consider particles. Take a curve $C$ embedded in $M$, and think of $C$ as the worldline of a particle. Suppose we can find a small 2-sphere $\Sigma$ in $M-C$ that is contractible in $M$ but not $M-C$. We can think of this 2-sphere as a 2-morphism $\Sigma: 1_{x} \Rightarrow 1_{x}$ in the path 2-groupoid of $M$. If we do $4 \mathrm{~d} B F$ theory on the spacetime $M-C$, the holonomy

$$
\operatorname{hol}(\Sigma) \in \mathfrak{s o}(3,1)
$$

will not change when we apply a homotopy to $\Sigma$. So, this holonomy describes some information about the particle-but so far as we know, the physical meaning of this information has not been worked out.

What if we had a field theory whose solutions were flat 2-connections for the Poincaré 2-group? Then we would have

$$
\operatorname{hol}(\Sigma) \in \mathbb{R}^{4}
$$

and there would be a tempting interpretation of this quantity: namely, as the energymomentum of our point particle. So, the puzzle posed at the end of the previous section is a tantalizing one. 
One may rightly ask if the 'strings' described above bear any relation to those of string theory. If they are merely surfaces cut out of spacetime, they lack the dynamical degrees of freedom normally associated to a string. Certainly they do not have an action proportional to their surface area, as for the Polyakov string. Indeed, one may ask if 'area' even makes sense in $4 \mathrm{~d} B F$ theory. After all, there is no metric on spacetime: the closest substitute is the $\mathfrak{s o}(3,1)$-valued 2 -form $B$.

Some of these problems may have solutions. For starters, when we remove a surface $X$ from our 4-manifold $M$, the action

$$
S(A, B)=\int_{M-X} \operatorname{tr}(B \wedge F)
$$

is no longer gauge-invariant: a gauge transformation changes the action by a boundary term which is an integral over $X$. We can remedy this by introducing fields that live on $X$, and adding a term to the action which is an integral over $X$ involving these fields. There are a number of ways to do this $[15,45,46,57,72]$. For some, the integral over $X$ is proportional to the area of the string worldsheet in the special case where the $B$ field arises from a cotetrad (that is, an $\mathbb{R}^{4}$-valued 1-form) as follows:

$$
B=e \wedge e
$$

where we use the isomorphism $\Lambda^{2} \mathbb{R}^{4} \cong \mathfrak{s o}(3,1)$. In this case there is close relation to the Nambu-Goto string, which has been carefully examined by Fairbairn et al. [46].

This is especially intriguing because when $B$ takes the above form, the $B F$ action becomes the usual Palatini action for general relativity:

$$
S(A, e)=\int_{M} \operatorname{tr}(e \wedge e \wedge F)
$$

where 'tr' is a suitable nondegenerate bilinear form on $\mathfrak{s o}(3,1)$. Unfortunately, solutions of Palatini gravity typically fail to obey the condition $t(B)=F$ when we take $B=e \wedge e$. So, we cannot construct 2-connections in the sense of Theorem 3 from these solutions! If we want to treat general relativity in 4 dimensions as a higher gauge theory, we need other ideas. We describe two possibilities at the end of Sect. 4.5.

\subsection{Inner automorphism 2-groups}

There is also a Lie 2-group where:

- $G$ is any Lie group,

- $H=G$,

- $t$ is the identity map,

- $\alpha$ is conjugation:

$$
\alpha(g) h=g h g^{-1} .
$$


Following Roberts and Schreiber [79] we call this the inner automorphism 2-group of $G$, and denote it by $\mathcal{I N \mathcal { N }}(G)$. We explain this terminology in the next section.

A 2-connection on the trivial $\mathcal{I N \mathcal { N }}(G)$-2-bundle over a manifold consists of a $\mathfrak{g}$-valued 1-form $A$ and a $\mathfrak{g}$-valued 2-form $B$ such that

$$
B=F
$$

since $t$ is now the identity. Intriguingly, 2-connections of this sort show up as solutions of a slight variant of $4 \mathrm{~d} B F$ theory. In a move that he later called his biggest blunder, Einstein took general relativity and threw an extra term into the equations: a 'cosmological constant' term, which gives the vacuum nonzero energy. We can do the same for topological gravity, or indeed $4 \mathrm{~d} B F$ theory for any group $G$. After all, what counts as a blunder for Einstein might count as a good idea for lesser mortals such as ourselves.

So, fix a 4-dimensional oriented manifold $M$ as our spacetime. As in ordinary $B F$ theory, take the fields to be a connection $A$ on the trivial principal $G$-bundle over $M$, together with a $\mathfrak{g}$-valued 2 -form $B$. The action for $B F$ theory 'with cosmological constant' is defined to be

$$
S(A, B)=\int_{M} \operatorname{tr}\left(B \wedge F-\frac{\lambda}{2} B \wedge B\right) .
$$

Setting the variation of the action equal to zero, we obtain these field equations:

$$
d B+[A, B]=0, \quad F=\lambda B .
$$

When $\lambda=0$, these are just the equations we saw in the previous section. But let us consider the case $\lambda \neq 0$. Then these equations have a drastically different character! The Bianchi identity $d F+[A, F]=0$, together with $F=\lambda B$, automatically implies that $d B+[A, B]=0$. So, to get a solution of this theory we simply take any connection $A$, compute its curvature $F$ and set $B=F / \lambda$.

This may seem boring: a field theory where any connection is a solution. But in fact it has an interesting relation to higher gauge theory. To see this, it helps to change variables and work with the field $\beta=\lambda B$. Then the field equations become

$$
d \beta+[A, \beta]=0, \quad F=\beta .
$$

Any solution of these equations gives a 2-connection on the trivial principal $\mathcal{I N \mathcal { N }}(G)$ 2-bundle over $M$ !

There is also a tantalizing relation to the cosmological constant in general relativity. If the $B$ field arises from a cotetrad as explained in the previous section:

$$
B=e \wedge e
$$


then the above action becomes

$$
S=\int_{M} \operatorname{tr}\left(e \wedge e \wedge F-\frac{\lambda}{2} e \wedge e \wedge e \wedge e\right) .
$$

When we choose the bilinear form 'tr' correctly, this is the action for general relativity with a cosmological constant proportional to $\lambda$.

There is some evidence [4] that $B F$ theory with nonzero cosmological constant can be quantized to obtain the so-called Crane-Yetter model [36,38], which is a spin foam model based on the category of representations of the quantum group associated to $G$. Indeed, in some circles this is taken almost as an article of faith. But a rigorous argument, or even a fully convincing argument, seems to be missing. So, this issue deserves more study.

The $\lambda \rightarrow 0$ limit of $B F$ theory is fascinating but highly singular, since for $\lambda \neq 0$ a solution is just a connection $A$, while for $\lambda=0$ a solution is a flat connection $A$ together with a $B$ field such that $d B+[A, B]=0$. At least in some rough intuitive sense, as $\lambda \rightarrow 0$ the group $H$ in the crossed module corresponding to $\mathcal{I N \mathcal { N }}(G)$ 'expands and flattens out' from the group $G$ to its tangent space $\mathfrak{g}$. Thus, $\mathcal{I N \mathcal { N }}(G)$ degenerates to the tangent 2-group $\mathcal{T} G$. It would be nice to make this precise using a 2-group version of the theory of group contractions.

\subsection{Automorphism 2-groups}

The inner automorphism group of the previous section is closely related to the automorphism 2-group $\mathcal{A U T}(H)$, defined using the crossed module where:

- $G=\operatorname{Aut}(H)$,

- $H$ is any Lie group,

- $t: H \rightarrow \operatorname{Aut}(H)$ sends any group element to the operation of conjugating by that element,

- $\alpha: \operatorname{Aut}(H) \rightarrow \operatorname{Aut}(H)$ is the identity.

We use the term 'automorphism 2-group' because $\mathcal{A U} \mathcal{T}(H)$ really is the 2-group of symmetries of $H$. Lie groups form a 2-category, any object in a 2-category has a 2group of symmetries, and the 2-group of symmetries of $H$ is naturally a Lie 2-group, which is none other than $\mathcal{A U} \mathcal{T}(H)$. See [14] for details.

A principal $\mathcal{A U} \mathcal{T}(H)$-2-bundle is usually called a nonabelian gerbe [27]. Nonabelian gerbes are a major test case for ideas in higher gauge theory. Indeed, almost the whole formalism of 2-connections was worked out first for nonabelian gerbes by Breen and Messing [29]. The one aspect they did not consider is the one we have focused on here: parallel transport. Thus, they did not impose the equation $\underline{t}(B)=F$, which we need to obtain holonomies satisfying the conditions of Theorem 3. Nonetheless, the quantity $F-\underline{t}(B)$ plays an important role in Breen and Messing's formalism: they call it the fake curvature. Generalizing their ideas slightly, for any Lie 2-group $\mathcal{G}$, we may define a connection on a trivial principal $\mathcal{G}$-2-bundle to be a pair consisting of a $\mathfrak{g}$-valued 1-form $A$ and an $\mathfrak{h}$-valued 2-form. A 2-connection is then a connection with vanishing fake curvature. 
The relation between the automorphism 2-group and the inner automorphism 2group is nicely explained in the work of Roberts and Schreiber [79]. As they discuss, for any group $G$ there is an exact sequence of 2-groups

$$
1 \rightarrow \mathcal{Z}(G) \rightarrow \mathcal{I N N}(G) \rightarrow \mathcal{A U} \mathcal{T}(G) \rightarrow \mathcal{O U} \mathcal{T}(G) \rightarrow 1
$$

where $\mathcal{Z}(G)$ is the center of $G$ and $\mathcal{O U T}(G)$ is the group of outer automorphisms of $G$, both regarded as 2-groups with only identity 2-morphisms.

Roberts and Schreiber go on to consider an analogous sequence of 3-groups constructed starting from a 2-group. Among these, the 'inner automorphism 3-group' $\mathcal{I N} \mathcal{N}(\mathcal{G})$ of a 2 -group $\mathcal{G}$ plays a special role [86]. The reason is that any connection on a principal $\mathcal{G}$-2-bundle, not necessarily obeying $\underline{t}(B)=F$, gives a flat 3 -connection on a principal $\mathcal{I N \mathcal { N }}(\mathcal{G})$-3-bundle! This in turn allows us to define a version of parallel transport for particles, strings and 2-branes.

This may give a way to understand general relativity in terms of higher gauge theory. As we have already seen in Sect. 4.3, Palatini gravity in $4 \mathrm{~d}$ spacetime involves an $\mathfrak{s o}(3,1)$-valued 1-form $A$ and an $\mathfrak{s o}(3,1)$-valued 2-form $B=e \wedge e$. This is precisely the data we expect for a connection on a principal $\mathcal{G}$-2-bundle where $\mathcal{G}$ is the tangent 2-group of the Lorentz group. Typically this connection fails to obey the equation $t(B)=F$. So, it is not a 2-connection. But, it gives a flat 3-connection on an $\mathcal{I} \mathcal{N} \mathcal{N}(\mathcal{T S O}(3,1))$-3-bundle. So, we may optimistically call $\mathcal{I N \mathcal { N }}(\mathcal{T S O}(3,1))$ the gravity 3-group.

Does the gravity 3-group actually shed any light on general relativity? The work of Martins and Picken [69] establishes a useful framework for studying these issues. They define a path 3-groupoid $\mathcal{P}_{3}(M)$ for a smooth manifold $M$. Given a Lie 3-group $\mathbf{G}$, they describe 3-connections on the trivial $\mathbf{G}$-3-bundle over $M$ as 3-functors

$$
\text { hol: } \mathcal{P}_{3}(M) \rightarrow \mathbf{G}
$$

Moreover, they show how to construct these functors from a 1-form, a 2-form, and a 3-form taking values in three Lie algebras associated to $\mathbf{G}$. In the case where $\mathbf{G}=$ $\mathcal{I N \mathcal { N }}(\mathcal{T S O}(3,1))$ and hol is a flat 3 -connection, this data reduces to an $\mathfrak{s o}(3,1)$ valued 1-form $A$ and an $\mathfrak{s o}(3,1)$-valued 2 -form $B$.

\subsection{String 2-groups}

The Lie 2-groups discussed so far are easy to construct. The string 2-group is considerably more subtle. Ultimately it forces upon us a deeper conception of what a Lie 2-group really is, and a more sophisticated approach to higher gauge theory. Treated in proper detail, these topics would carry us far beyond the limits of this quick introduction. But it would be a shame not to mention them at all.

Suppose we have a central extension of a Lie group $G$ by an abelian Lie group $A$. In other words, suppose we have a short exact sequence of Lie groups

$$
1 \rightarrow A \rightarrow H \stackrel{t}{\rightarrow} G \rightarrow 1
$$


where the image of $A$ lies in the center of $H$. Then we can construct an action $\alpha$ of $G$ on $H$ as follows. The map $t: H \rightarrow G$ describes $H$ as a fiber bundle over $G$, so choose a section of this bundle: that is, a function $s: G \rightarrow H$ with $t(s(g))=g$, not necessarily a homomorphism. Then set

$$
\alpha(g) h=s(g) h s(g)^{-1} .
$$

Since $A$ is included in the center of $H, \alpha$ is independent of the choice of $s$. Thanks to this, we do not need a global smooth section $s$ to check that $\alpha(g)$ depends smoothly on $g$ : it suffices that there exist a local smooth section in a neighborhood of each $g \in G$, and indeed this is always true. We can use these local sections to define $\alpha$ globally, since they must give the same $\alpha$ on overlapping neighborhoods.

Given all this, we can check that $t$ is $G$-equivariant and that the Peiffer identity holds. So, we obtain a Lie 2-group where:

- $G$ is any Lie group,

- $H$ is any Lie group,

- $t: H \rightarrow G$ makes $H$ into a central extension of $G$,

- $\alpha$ is given by $\alpha(g) h=s(g) h s(g)^{-1}$ where $s: G \rightarrow H$ is any section.

We call this the central extension 2-group $\mathcal{C}(H \stackrel{t}{\rightarrow} G)$.

To get concrete examples, we need examples of central extensions. For any choice of $G$ and $A$, we can always take $H=G \times A$ and use the 'trivial' central extension

$$
1 \rightarrow A \rightarrow A \times G \rightarrow G \rightarrow 1 .
$$

For more interesting examples, we need nontrivial central extensions. These tend to arise from problems in quantization. For example, suppose $V$ is a finite-dimensional symplectic vector space: that is, a vector space equipped with a nondegenerate antisymmetric bilinear form

$$
\omega: V \times V \rightarrow \mathbb{R}
$$

Then we can make $H=V \times \mathbb{R}$ into a Lie group called the Heisenberg group, with the product

$$
(u, a)(v, b)=(u+v, a+b+\omega(u, v)) .
$$

The Heisenberg group plays a fundamental role in quantum mechanics, because we can think of $V$ as the phase space of a classical point particle. If we let $G$ stand for $V$ regarded as an abelian Lie group, then elements of $G$ describe translations in phase space: that is, translations of both position and momentum. The Heisenberg group $H$ describes how these translations commute only 'up to a phase' when we take quantum mechanics into account: the phase is given by $\exp (i \omega(u, v))$. There is a homomorphism $t: H \rightarrow G$ that forgets this phase information, given by

$$
t(u, a)=u
$$


This exhibits $H$ as a central extension of $G$. We thus obtain a central extension 2-group $\mathcal{C}(H \stackrel{t}{\longrightarrow} G)$, called the Heisenberg 2-group of the symplectic vector space $V$.

The applications of Heisenberg 2-groups seem largely unexplored, and should be worth studying. So far, much more work has been put into understanding 2-groups arising from central extensions of loop groups. The reason is that central extensions of loop groups play a basic role in string theory and conformal field theory, as nicely explained by Pressley and Segal [78].

Suppose that $G$ is a connected and simply-connected compact simple Lie group $G$. Define the loop group $\Omega G$ to be the set of all smooth paths $\gamma:[0,1] \rightarrow G$ that start and end at the identity of $G$. This becomes a group under pointwise multiplication, and in fact it is a kind of infinite-dimensional Lie group [70].

For each integer $k$, called the level, the loop group has a central extension

$$
1 \rightarrow \mathrm{U}(1) \rightarrow \widehat{\Omega_{k} G} \stackrel{t}{\rightarrow} \Omega G \rightarrow 1 .
$$

These extensions are all different, and all nontrivial except for $k=0$. In physics, they arise because the $2 \mathrm{~d}$ gauge theory called the Wess-Zumino-Witten model has an 'anomaly'. The loop group $\Omega G$ acts as gauge transformations in the classical version of this theory. However, when we quantize the theory, we obtain a representation of $\Omega G$ only 'up to a phase'- that is, a projective representation. This can be understood as an honest representation of the central extension $\widehat{\Omega_{k} G}$, where the integer $k$ appears in the Lagrangian for the Wess-Zumino-Witten model.

Starting from this central extension we can construct a central extension 2-group called the level-k loop 2-group of $G, \mathcal{L}_{k}(G)$. This is an infinite-dimensional Lie 2-group, meaning that it comes from a crossed module where the groups involved are infinite-dimensional Lie groups, and all the maps are smooth. Moreover, it fits into an exact sequence

$$
1 \rightarrow \mathcal{L}_{k}(G) \rightarrow \mathcal{S} \mathcal{T} \mathcal{R} \mathcal{I N} \mathcal{G}_{k}(G) \longrightarrow G \rightarrow 1
$$

where the middle term, the level-k string 2-group of $G$, has very interesting properties [9].

Since the string 2-group $\mathcal{S} \mathcal{T} \mathcal{R} \mathcal{I} \mathcal{G G}_{k}(G)$ is an infinite-dimensional Lie 2-group, it is a topological 2-group. There is a way to take any topological 2-group and squash it down to a topological group [9,19]. Applying this trick to $\mathcal{S} \mathcal{T} \mathcal{R} \mathcal{I} \mathcal{G}_{k}(G)$ when $k=1$, we obtain a topological group whose homotopy groups match those of $G$ except for the third homotopy group, which has been made trivial. In the special case where $G=\operatorname{Spin}(n)$, this topological group is called the 'string group', since to consistently define superstrings propagating on a spin manifold, we must reduce its structure group from $\operatorname{Spin}(n)$ to this group [96]. The string group also plays a role in Stolz and Teichner's work on elliptic cohomology, which involves a notion of parallel transport over surfaces [91]. There is a lot of sophisticated mathematics involved here, but ultimately much of it should arise from the way string 2-groups are involved in the parallel transport of strings! The work of Sati et al. [83] provides good evidence for this, as does the work of Waldorf [92]. 
In fact, the string Lie 2-group had lived through many previous incarnations before being constructed as an infinite-dimensional Lie 2-group. Brylinski and McLaughlin $[32,33]$ thought of it as a U(1) gerbe over the group $G$. The fact that this gerbe is 'multiplicative' makes it something like a group in its own right [31]. This viewpoint was also been explored by Murray and Stevenson [75].

Later, Baez and Crans [8] constructed a Lie 2-algebra $\mathfrak{s t r i n g}_{k}(\mathfrak{g})$ corresponding to the string Lie 2-group. For pedagogical purposes, our discussion of Lie 2-groups has focused solely on 'strict' 2-groups, where the 1-morphisms satisfy the group axioms strictly, as equations. However, there is also an extensive theory of 'weak' 2-groups, where the 1-morphisms obey the group axioms only up to invertible 2-morphisms [14]. Following this line of thought, we may also define weak Lie 2-algebras [81], and the Lie 2-algebra $\mathfrak{s t r i n g}_{k}(\mathfrak{g})$ is one of these where only the Jacobi identity fails to hold strictly.

The beauty of weak Lie 2-algebras is that $\mathfrak{s t r i n g}_{k}(\mathfrak{g})$ is very easy to describe in these terms. In particular, it is finite-dimensional. The hard part is constructing a weak Lie 2-group corresponding to this weak Lie 2-algebra. It is easy to check that any strict Lie 2-algebra has a corresponding strict Lie 2-group. Weak Lie 2-algebras are more tricky [14]. Baez et al. [9] dodged this problem by showing that the string Lie 2-algebra is equivalent (in some precise sense) to a strict Lie 2-algebra, which however is infinite-dimensional. They then constructed the infinite-dimensional strict Lie 2-group corresponding to this strict Lie 2-algebra. This is just $\mathcal{S T}_{\mathcal{T}} \mathcal{I N G}_{k}(G)$ as described above.

On the other hand, a finite-dimensional model of the string 2-group was recently introduced by Schommer-Pries [85]. This uses an improved definition of 'weak Lie 2-group', based on an important realization: the correct maps between smooth groupoids are not the smooth functors, but something more general [62]. We have already mentioned this in our discussion of connection: a smooth functor hol: $\mathcal{P}_{1}(M) \rightarrow G$ is a connection on the trivial principal $G$-bundle over $M$, while one of these more general maps is a connection on an arbitrary principal $G$-bundle over $M$. If we take this lesson to heart, we are led into the world of 'stacks' - and in that world, we can find a finite-dimensional version of the string 2-group.

There has also been progress on constructing weak Lie $n$-groups from weak Lie $n$-algebras for $n>2$. Getzler [54] and Henriques [59] have developed an approach that works for all $n$, even $n=\infty$. Their approach is able to handle weak Lie $\infty$-algebras of a sort known as ' $L_{\infty}$-algebras'. Quite roughly, the idea is that in an $L_{\infty}$-algebra, the Jacobi identity holds only weakly, while the antisymmetry of the bracket still holds strictly.

In fact, $L_{\infty}$-algebras were developed by Stasheff and collaborators $[66,84]$ before higher gauge theory became recognized as a subject of study. But more recently, Sati et al. [82,83] have developed a lot of higher gauge theory with the help of $L_{\infty}$ algebras. Together with the insights of Aschieri et al. [1,2], their work makes it clear that superstring theory, supergravity and even the mysterious ' $M$-theory' have strong ties to higher gauge theory. For example, 11-dimensional supergravity can be seen as a higher gauge theory governed by a certain 'Lie 3-superalgebra' which they call $\mathfrak{s u g r a}(10,1)$. The number 3 here relates to the 2-brane solutions of 11-dimensional supergravity: just as parallel transport of strings is described by 2-connections, the 
parallel transport of 2-branes is described by 3-connections, which in the supersymmetric case involve Lie 3 -superalgebras.

In fact, $\mathfrak{s u g r a}(10,1)$ is one of a family of four Lie 3 -superalgebras that extend the Poincaré Lie superalgebra in dimensions 4, 5, 7 and 11. These can be built via a systematic construction starting from the four normed division algebras: the real numbers, the complex numbers, the quaternions and the octonions [12]. These four algebras also give rise to Lie 2-superalgebras extending the Poincaré Lie superalgebra in dimensions 3, 4, 6, and 10. The Lie 2-superalgebras are related to superstring theories in dimensions 3, 4, 6, and 10, while the Lie 3-superalgebras are related to super-2-brane theories in dimensions 4, 5, 7 and 11. All these theories, and even their relation to division algebras, have been known since the late 1980s [42]. Higher gauge theory provides new insights into the geometry of these theories. In particular, the work of Castellani etal. [35] can be seen as implicitly making extensive use of Lie $n$-superalgebras - but this only became clear later, through the work of Sati et al. [83].

Alas, explaining these fascinating issues in detail would vastly expand the scope of this paper. We should instead return to simpler things: gauge transformations, curvature, and nontrivial 2-bundles.

\section{Further topics}

So far our introduction to higher gauge theory has neglected the most important topic of all: gauge transformations! We have also said nothing about curvature or nontrivial 2-bundles. Now it is time to begin correcting these oversights.

\subsection{Gauge transformations}

First consider ordinary gauge theory. Suppose that $M$ is a manifold and $G$ is a Lie group. Then a gauge transformation on the trivial principal $G$-bundle over $M$ simply amounts to a smooth function

$$
g: M \rightarrow G
$$

while a connection on this bundle can be seen as a $\mathfrak{g}$-valued 1-form. A gauge transformation $g$ acts on a connection $A$ to give a new connection $A^{\prime}$ as follows:

$$
A^{\prime}=g A g^{-1}+g d g^{-1} \text {. }
$$

This formula makes literal sense if $G$ is a group of matrices: then $\mathfrak{g}$ also consists of matrices, so we can freely multiply elements of $G$ with elements of $\mathfrak{g}$. If $G$ is an arbitrary Lie group the formula requires a bit more careful interpretation, but it still makes sense. A well-known calculation says the curvature $F^{\prime}=d A^{\prime}+A^{\prime} \wedge A^{\prime}$ of the gauge-transformed connection is just the curvature of the original connection conjugated by $g$ :

$$
F^{\prime}=g F g^{-1} \text {. }
$$


In higher gauge theory the formulas are similar, but a bit more complicated. Suppose $M$ is a manifold and $\mathcal{G}$ is a Lie 2-group with crossed module $(G, H, t, \alpha)$. It will be helpful to take everything in this crossed module and differentiate it. Doing this, we get:

- the Lie algebra $\mathfrak{g}$ of $G$,

- the Lie algebra $\mathfrak{h}$ of $H$,

- the Lie algebra homomorphism $\underline{t}: \mathfrak{h} \rightarrow \mathfrak{g}$ obtained by differentiating $t: H \rightarrow G$, and

- the Lie algebra homomorphism $\underline{\alpha}: \mathfrak{g} \rightarrow \mathfrak{a u t}(H)$ obtained by differentiating $\alpha: G \rightarrow \operatorname{Aut}(H)$.

Here $\operatorname{aut}(H)$ is the Lie algebra of $\operatorname{Aut}(H)$. It is best to think of this as the Lie algebra of derivations of $\mathfrak{h}$ : that is, linear maps $D: \mathfrak{h} \rightarrow \mathfrak{h}$ such that

$$
D[x, y]=[D x, y]+[x, D y] .
$$

If we differentiate the two equations in the definition of a crossed module, we obtain the $\mathfrak{g}$-equivariance of $\underline{t}$ :

$$
\underline{t}(\underline{\alpha}(x)(y))=[x, \underline{t}(y)]
$$

and the infinitesimal Peiffer identity:

$$
\underline{\alpha}(\underline{t}(y))\left(y^{\prime}\right)=\left[y, y^{\prime}\right]
$$

where $x \in \mathfrak{g}$ and $y, y^{\prime} \in \mathfrak{h}$. In case the reader is curious: we write $\underline{t}$ and $\underline{\alpha}$ instead of $d t$ and $d \alpha$ because later we will do computations involving these maps and also differential forms, where $d$ stands for the exterior derivatve.

A quadruple $(\mathfrak{g}, \mathfrak{h}, \underline{t}, \underline{\alpha})$ of Lie algebras and homomorphisms obeying these two equations is called an infinitesimal crossed module. Just as crossed modules are a way of working with 2-groups, infinitesimal crossed modules are a way of working with Lie 2-algebras [8]. Any infinitesimal crossed module comes from a Lie 2-group, and this Lie 2-group is unique if we demand that $G$ and $H$ be connected and simply connected.

But we digress! We have introduced infinitesimal crossed modules in order to say how gauge transformations act on 2-connections. A gauge transformation of the trivial $\mathcal{G}$-2-bundle over $M$ consists of two pieces of data:

- a smooth function $g: M \rightarrow G$,

- an $\mathfrak{h}$-valued 1-form $a$ on $M$.

Why two pieces of data? Perhaps this should not be so surprising. Remember, a 2-connection also consists of two pieces of data:

- a $\mathfrak{g}$-valued 1-form $A$ on $M$,

- an $\mathfrak{h}$-valued 2-form $B$ on $M$ satisfying $\underline{t}(B)=F$. 
Breen and Messing [29] worked out how gauge transformations act on connections on nonabelian gerbes, and their work was later generalized to 2-connections on arbitrary principal 2-bundles $[17,88,89]$. Here we merely present the formulas. A gauge transformation $(g, a)$ acts on a 2-connection $(A, B)$ to give a new 2-connection $\left(A^{\prime}, B^{\prime}\right)$ as follows:

$$
\begin{aligned}
& A^{\prime}=g A g^{-1}+g d g^{-1}+\underline{t}(a) \\
& B^{\prime}=\alpha(g)(B)+\underline{\alpha}\left(A^{\prime}\right) \wedge a+d a+a \wedge a
\end{aligned}
$$

The second formula requires a bit of explanation. In the first term we compose $\alpha: G \rightarrow$ $\operatorname{Aut}(H)$ with $g: M \rightarrow G$ and obtain an $\operatorname{Aut}(H)$-valued function $\alpha(g)$, which then acts on the $\mathfrak{h}$-valued 2-form $B$ to give a new $\mathfrak{h}$-valued 2-form $\alpha(g)(B)$. In the second term we start by composing $A^{\prime}$ with $\underline{\alpha}$ to obtain an $\mathfrak{a u t}(H)$-valued 1-form $\underline{\alpha}\left(A^{\prime}\right)$. Then we wedge this with $a$, letting $\mathfrak{a u t}(H)$ act on $\mathfrak{h}$ as part of this process, and obtain an $\mathfrak{h}$-valued 2-form.

As a kind of consistency check and test of our understanding, let us see why the gauge-transformed 2-connection $\left(A^{\prime}, B^{\prime}\right)$ satisfies the equation $\underline{t}\left(B^{\prime}\right)=F^{\prime}$. First let us compute the curvature 2-form $F^{\prime}$ of the gauge-transformed 2-connection:

$$
\begin{aligned}
F^{\prime}= & d A^{\prime}+A^{\prime} \wedge A^{\prime} \\
= & d\left(g A g^{-1}+g d g^{-1}+\underline{t}(a)\right) \\
& +\left(g A g^{-1}+g d g^{-1}+\underline{t}(a)\right) \wedge\left(g A g^{-1}+g d g^{-1}+\underline{t}(a)\right)
\end{aligned}
$$

This looks like a mess-but except for the terms containing $\underline{t}(a)$, this is just the usual mess we get in ordinary gauge theory when we compute the curvature of a gauge transformed connection. So, we have:

$$
\begin{aligned}
F^{\prime} & =g F g^{-1}+d(\underline{t}(a))+\underline{t}(a) \wedge A^{\prime}+A^{\prime} \wedge \underline{t}(a) \\
& =g F g^{-1}+\underline{t}(d a)+\left[\underline{t}(a), A^{\prime}\right]
\end{aligned}
$$

where we use the fact that $d(\underline{t}(a))=\underline{t}(d a)$ and rewrite $A^{\prime} \wedge \underline{t}(a)+\underline{t}(a) \wedge A^{\prime}$ as a graded commutator.

On the other hand, we have

$$
\underline{t}\left(B^{\prime}\right)=\underline{t}(\alpha(g)(B))+\underline{t}\left(\underline{\alpha}\left(A^{\prime}\right) \wedge a\right)+\underline{t}(d a+a \wedge a)
$$

The $G$-equivariance of $t$ implies that $t(\alpha(g)(B))=g \underline{t}(B) g^{-1}$, and the $\mathfrak{g}$-equivariance of $\underline{t}$ implies that $\left.\underline{t}\left(\underline{\alpha}\left(A^{\prime}\right)\right) \wedge a\right)=\left[A^{\prime}, \underline{t}(a)\right]$. So, we see that

$$
\underline{t}\left(B^{\prime}\right)=g \underline{t}(B) g^{-1}+\left[A^{\prime}, d t(a)\right]+\underline{t}(d a+a \wedge a)
$$

and thus $\underline{t}\left(B^{\prime}\right)=F^{\prime}$, as desired. 


\subsection{Curvature}

Suppose $\mathcal{G}$ is a Lie 2-group whose crossed module is $(G, H, t, \alpha)$, and let $(\mathfrak{g}, \mathfrak{h}, \underline{t}, \underline{\alpha})$ be the corresponding differential crossed module. Suppose we have a connection on the trivial $\mathcal{G}$-2-bundle over $M$ : that is, a $\mathfrak{g}$-valued 1 -form $A$ and an $\mathfrak{h}$-valued 2-form $B$.

As in ordinary gauge theory, we may define the curvature of this connection to be the $\mathfrak{g}$-valued 2-form given by:

$$
F=d A+A \wedge A
$$

We also have another $\mathfrak{g}$-valued 2 -form, the fake curvature $F-t \underline{t}(B)$. Recall from Sect. 3 that only a connection with vanishing fake curvature counts as a 2 -connection. In other words, we need $t(B)=F$ to obtain well-defined parallel transport over surfaces.

We may also define the 2-curvature of a connection in higher gauge theory. This is the $\mathfrak{h}$-valued 3 -form given by:

$$
Z=d B+\underline{\alpha}(A) \wedge B .
$$

In the second term here, we compose $\underline{\alpha}: \mathfrak{g} \rightarrow \mathfrak{a u t}(H)$ with the $\mathfrak{g}$-valued 1-form $A$ and obtain an $\mathfrak{a u t}(H)$-valued function $\underline{\alpha}(g)$. Then we wedge this with $B$, letting $\mathfrak{a u t}(H)$ act on $\mathfrak{h}$ as part of this process, and obtain an $\mathfrak{h}$-valued 2 -form.

The intuitive idea of 2-curvature is this: just as the curvature describes the holonomy of a connection around an infinitesimal loop, the 2-curvature describes the holonomy of a 2-connection over an infinitesimal 2-sphere. This can be made precise using formulas for holonomies over surfaces [67,68,88,89]

If the 2-curvature of a 2-connection vanishes, the holonomy over a surface will not change if we apply a smooth homotopy to that surface while keeping its edges fixed. A 2-connection whose curvature and 2-curvature both vanish truly deserves to be called flat. We have seen flat 2-connections already in our discussion of 4-dimensional $B F$ theory in Sect. 4.3: the solutions of this theory are flat 2-connections.

\subsection{Nontrivial 2-bundles}

So far we have implicitly been looking at 2-connections on trivial 2-bundles. This is fine locally. But there are also interesting issues involving nontrivial 2-bundles, which become crucial when we work globally.

A careful treatment of 2-bundles would require some work, and the reader interested in this topic would do well to start with Moerdijk's introductory paper on 'stacks' and 'gerbes' [71]. Here we take a less sophisticated approach: we simply describe how to build a principal 2-bundle and put a 2-connection on it. Since we do not say when two principal 2-bundles built this way are 'same', our treatment is incomplete. The reader can find more details elsewhere [19,20,26-29,88,89]. We warn the reader that almost every paper in the literature uses different notation, sign conventions, and so forth.

First recall ordinary gauge theory: suppose $G$ is a Lie group and $M$ a manifold. In this case we can build a principal $G$-bundle over $M$ using transition functions. 
First, write $M$ as the union of open sets or patches $U_{i} \subseteq M$ :

$$
M=\bigcup_{i} U_{i}
$$

Then, choose a smooth transition function on each double intersection of patches:

$$
g_{i j}: U_{i} \cap U_{j} \rightarrow G
$$

These transition functions give gauge transformations. We can build a principal $G$-bundle over all of $M$ by gluing together trivial bundles over the patches with the help of these gauge transformations. However, this procedure will only succeed if the transition functions satisfy a consistency condition on each triple intersection:

$$
g_{i j}(x) g_{j k}(x)=g_{i k}(x)
$$

for all $x \in U_{i} \cap U_{j} \cap U_{k}$. This equation is called a cocycle condition. We can visualize it as a triangle:

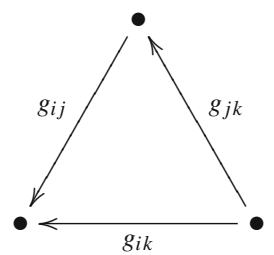

where we suppress the variable $x$ for the sake of readability. The idea is that this triangle should 'commute': the direct way of identifying points in the trivial bundle in the $i$ th patch to points in the trivial bundle over the $k$ th patch should match the indirect way which proceeds via the $j$ th patch.

A similar but more elaborate recipe works for higher gauge theory. Now let $\mathcal{G}$ be a Lie 2-group with crossed module $(G, H, t, \alpha)$. To build a $\mathcal{G}$-2-bundle, we start by choosing transition functions on double intersections of patches:

$$
g_{i j}: U_{i} \cap U_{j} \rightarrow G
$$

However, now it makes sense to replace the equation in the cocycle condition by a 2-morphism! So, for each triple intersection we choose 2-morphisms in $\mathcal{G}$ :

$$
\gamma_{i j k}(x): g_{i j}(x) g_{j k}(x) \Rightarrow g_{i k}(x)
$$

depending smoothly on $x \in U_{i} \cap U_{j} \cap U_{k}$. We can again visualize these as triangles: 


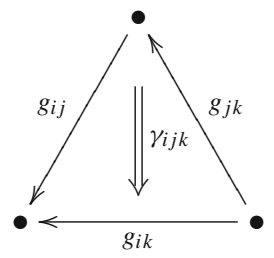

But now we demand that these 2-morphisms themselves obey a cocycle condition on quadruple intersections of patches. As we ascend the ladder of higher gauge theory, triangles become tetrahedra and then higher-dimensional simplexes. In this case, the cocycle condition says that this tetrahedron commutes:

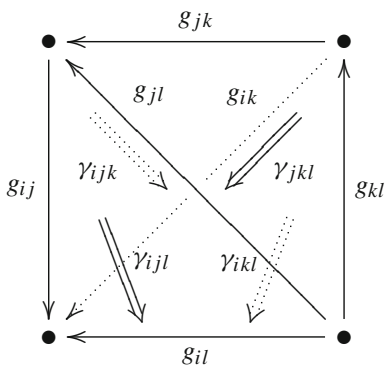

By saying that this tetrahedron 'commutes', we mean that the composite of the front two sides equals the composite of the back two sides:

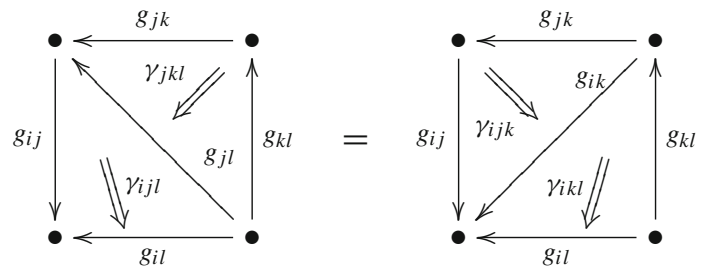

We need whiskering to compose the 2-morphisms in this diagram, as explained near the end of Sect. 3. So in equations, the tetrahedral cocycle condition says that:

$$
\gamma_{i j l} \cdot\left(g_{i j} \circ \gamma_{j k l}\right)=\left(\gamma_{i j k} \circ g_{k l}\right) \cdot \gamma_{i k l}
$$

where - stands for vertical composition and o stands for whiskering.

We can describe this cocycle condition in a more down-to-earth manner if we use Theorem 2, which says that a 2-morphism

$$
\gamma_{i j k}: g_{i j} g_{j k} \Rightarrow g_{i k}
$$


is the same as an element $h_{i j k} \in H$ such that

$$
t\left(h_{i j k}\right) g_{i j} g_{j k}=g_{i k} .
$$

This theorem also gives formulas for vertical and horizontal composition in terms of the groups $G$ and $H$. Since whiskering by a morphism is horizontal composition with its identity 2-morphism, we can also express whiskering in these terms. So, a little calculation - a wonderful exercise for the would-be higher gauge theorist-shows that:

$$
h_{i j l} \alpha\left(g_{i j}\right)\left(h_{j k l}\right)=h_{i j k} h_{i k l}
$$

where $\alpha$ is the action of $G$ on $H$.

There is no need to have $g_{i i}=1$ in this formalism; we should really choose a 2-morphism from $g_{i i}$ to 1 . However, without loss of generality, we can assume that $g_{i i}=1$ and set this 2-morphism equal to the identity. We can also assume that $h_{i j k}=1$ whenever two or more of the indices $i, j, k$ are equal. The reason is that Bartels [26] has shown that any principal 2-bundle is equivalent to one for which these simplifying assumptions hold. We will make these assumptions in what follows. For the full story without these assumptions, see Schreiber and Waldorf [88,89].

Now consider connections. Again, it helps to begin by reviewing the story for ordinary gauge theory. Suppose we have a manifold $M$ written as a union of patches $U_{i}$, and suppose we have principal $G$-bundle over $M$ built using transition functions $g_{i j}$. To put a connection on this bundle, we first put a connection on the trivial bundle over each patch: that is, for each $i$, we choose a $\mathfrak{g}$-valued 1-form $A_{i}$ on $U_{i}$. But then we must check to see if these fit together to give a well-defined connection on all of $M$. For this, we need the gauge transform of the connection on the $j$ th patch to equal the connection on the $i$ th patch:

$$
A_{i}=g_{i j} A_{j} g_{i j}^{-1}+g_{i j} d g_{i j}^{-1}
$$

on each double intersection $U_{i} \cap U_{j}$.

The story is similar for 2-connections. Suppose we have a principal $\mathcal{G}$-2-bundle over $M$ built using transition functions $g_{i j}$ and $h_{i j k}$ as described above. To equip this 2-bundle with a 2-connection, we first put a 2-connection on the trivial 2-bundle over each patch. So, on each open set $U_{i}$ we choose a $\mathfrak{g}$-valued 1-form $A_{i}$ and an $\mathfrak{h}$-valued 2-form $B_{i}$ with $\underline{t}\left(B_{i}\right)=F_{i}$. But then we must fit these together to get a 2-connection on all of $M$.

For this, we should follow the ideas from Sect. 5.1 on how gauge transformations work in higher gauge theory. So, we choose an $\mathfrak{h}$-valued 1 -form $a_{i j}$ on each double intersection $U_{i} \cap U_{j}$, and require that

$$
\begin{aligned}
A_{i} & =g_{i j} A_{j} g_{i j}^{-1}+g_{i j} d g_{i j}^{-1}+\underline{t}\left(a_{i j}\right) \\
B_{i} & =\alpha\left(g_{i j}\right)\left(B_{j}\right)+\underline{\alpha}\left(A_{i}\right) \wedge a_{i j}+d a_{i j}+a_{i j} \wedge a_{i j} .
\end{aligned}
$$


These equations say that the 2-connection $\left(A_{i}, B_{i}\right)$ is a gauge-transformed version of $\left(A_{j}, B_{j}\right)$. The appearance of $A_{i}$ on the right-hand side of the second equation is not a typo! Finally, the 1 -forms $a_{i j}$ must obey a consistency condition on triple intersections:

$$
h_{i j k}^{-1} \underline{\alpha}\left(A_{i}\right)\left(h_{i j k}\right)+h_{i j k}^{-1} d h_{i j k}+\alpha\left(g_{i j}\right)\left(a_{j k}\right)+a_{i j}=h_{i j k}^{-1} a_{i k} h_{i j k}
$$

Where does this consistency condition come from? Indeed, what does it even mean? We have not yet defined ' $h{ }^{-1} \underline{\alpha}(A)(h)$ ' when $A \in \mathfrak{g}$ and $h$ is an element of the group $H$.

We could systematically derive this condition from a more conceptual approach to 2-connections $[17,88,89]$, but it will be marginally less stressful to motivate it as follows. For every triple intersection $U_{i} \cap U_{j} \cap U_{k}$ we have three equations relating $A_{i}, A_{j}$ and $A_{k}$ :

$$
\begin{aligned}
A_{i} & =g_{i j} A_{j} g_{i j}^{-1}+g_{i j} d g_{i j}^{-1}+\underline{t}\left(a_{i j}\right) \\
A_{j} & =g_{j k} A_{k} g_{j k}^{-1}+g_{j k} d g_{j k}^{-1}+\underline{t}\left(a_{j k}\right) \\
A_{k} & =g_{k i} A_{i} g_{k i}^{-1}+g_{k i} d g_{k i}^{-1}+\underline{t}\left(a_{k i}\right)
\end{aligned}
$$

The first equation expresses $A_{i}$ in terms of $A_{j}$. We can substitute the second equation in the first to get a formula for $A_{i}$ in terms of $A_{k}$. Then we can use the third equation to get a formula for $A_{i}$ in terms of itself! We would like to be able to simplify this formula to get simply $A_{i}=A_{i}$. The consistency condition, (2), ensures that we can do this.

This calculation is a bit of a workout; let us see how it goes. We begin by doing the substitutions:

$$
\begin{aligned}
A_{i}= & g_{i j} A_{j} g_{i j}^{-1}+g_{i j} d g_{i j}^{-1}+\underline{t}\left(a_{i j}\right) \\
= & g_{i j}\left(g_{j k} A_{k} g_{j k}^{-1}+g_{j k} d g_{j k}^{-1}+\underline{t}\left(a_{j k}\right)\right) g_{i j}^{-1}+g_{i j} d g_{i j}^{-1}+\underline{t}\left(a_{i j}\right) \\
= & g_{i j}\left(g_{j k}\left(g_{k i} A_{i} g_{k i}^{-1}+g_{k i} d g_{k i}^{-1}+\underline{t}\left(a_{k i}\right)\right) g_{j k}^{-1}+g_{j k} d g_{j k}^{-1}+\underline{t}\left(a_{j k}\right)\right) g_{i j}^{-1} \\
& +g_{i j} d g_{i j}^{-1}+\underline{t}\left(a_{i j}\right) .
\end{aligned}
$$

Then we do a bit of simplification:

$$
\begin{aligned}
A_{i}= & g_{i j} g_{j k} g_{k i} A_{i}\left(g_{i j} g_{j k} g_{k i}\right)^{-1}+g_{i j} g_{j k} g_{k i} d\left(g_{i j} g_{j k} g_{k i}\right)^{-1} \\
& +g_{i j} g_{j k} \underline{t}\left(a_{k i}\right)\left(g_{i j} g_{j k}\right)^{-1}+g_{i j} \underline{t}\left(a_{j k}\right) g_{i j}^{-1}+\underline{t}\left(a_{i j}\right) .
\end{aligned}
$$

Since $t\left(h_{i j k}\right) g_{i j} g_{j k}=g_{i k}$ and we are assuming that $g_{i k}^{-1}=g_{k i}$, we have

$$
g_{i j} g_{j k} g_{k i}=t\left(h_{i j k}\right)^{-1}
$$


so

$$
\begin{aligned}
0= & t\left(h_{i j k}\right)^{-1} A_{i} t\left(h_{i j k}\right)-A_{i}+t\left(h_{i j k}\right)^{-1} d t\left(h_{i j k}\right) \\
& +t\left(h_{i j k}\right)^{-1} g_{i k} \underline{t}\left(a_{k i}\right) g_{i k}^{-1} t\left(h_{i j k}\right)+g_{i j} \underline{t}\left(a_{j k}\right) g_{i j}^{-1}+\underline{t}\left(a_{i j}\right) .
\end{aligned}
$$

Now, if $G$ is a matrix group, we can freely multiply group elements and Lie algebra elements. Then for any $h \in H$ and $A \in \mathfrak{g}$ we have

$$
t(h)^{-1} A t(h)-A=t(h)^{-1}[A, t(h)] .
$$

This will allow us to simplify the first two terms in (3). Moreover, for any $g \in G$, $h \in H$ we have

$$
t\left(h^{-1} \alpha(g) h\right)=t(h)^{-1} t(\alpha(g)(h))=t(h)^{-1} g t(h) g^{-1} .
$$

Taking $g=\exp (s A)$ for $A \in \mathfrak{g}$ and differentiating this equation with respect to $s$ at $s=0$, we get

$$
\underline{t}\left(h^{-1} \underline{\alpha}(A)(h)\right)=t(h)^{-1} A t(h)-A
$$

where on the left side $\underline{\alpha}$ means the derivative of the map $\alpha: G \times H \rightarrow H$ with respect to its first argument, while $t$ is the derivative of $t$. Here are we extending our previous definitions of $\underline{\alpha}$ and $\underline{t}$. Combining (4) and (5) we see that

$$
t(h)^{-1}[A, t(h)]=d t\left(h^{-1} d \alpha(A)(h)\right) .
$$

In fact this result holds even when $G$ is a non-matrix Lie group, as long as we carefully make sense of both sides.

Using this result, we can rewrite (3) as follows:

$$
\begin{aligned}
0= & \underline{t}\left(h_{i j k}^{-1} \underline{\alpha}\left(A_{i}\right)\left(h_{i j k}\right)\right)+t\left(h_{i j k}\right)^{-1} d t\left(h_{i j k}\right) \\
& +t\left(h_{i j k}\right)^{-1} g_{i k} \underline{t}\left(a_{k i}\right) g_{i k}^{-1} t\left(h_{i j k}\right)+g_{i j} \underline{t}\left(a_{j k}\right) g_{i j}^{-1}+\underline{t}\left(a_{i j}\right)
\end{aligned}
$$

Each term in the above equation is $\underline{t}$ applied to an $\mathfrak{h}$-valued 1-form. Writing down all these $\mathfrak{h}$-valued 1 -forms, we see that the above equation will be true if this condition holds:

$$
\begin{aligned}
0= & h_{i j k}^{-1} \underline{\alpha}\left(A_{i}\right)\left(h_{i j k}\right)+h_{i j k}^{-1} d h_{i j k} \\
& +h_{i j k}^{-1} \alpha\left(g_{i k}\right)\left(a_{k i}\right) h_{i j k}+\alpha\left(g_{i j}\right)\left(a_{j k}\right)+a_{i j}
\end{aligned}
$$

This is our consistency condition in disguise! To remove the disguise, let us simplify it a bit further. When $i=j$ this condition reduces to

$$
a_{k i}+\alpha\left(g_{i k}\right)\left(a_{k i}\right)=0 .
$$


Reinserting this result we obtain

$$
0=h_{i j k}^{-1} \underline{\alpha}\left(A_{i}\right)\left(h_{i j k}\right)+h_{i j k}^{-1} d h_{i j k}-h_{i j k}^{-1} a_{i k} h_{i j k}+\alpha\left(g_{i j}\right)\left(a_{j k}\right)+a_{i j}
$$

Voilà! This is clearly equivalent to the consistency condition we stated in the first place, (2):

$$
h_{i j k}^{-1} \underline{\alpha}\left(A_{i}\right)\left(h_{i j k}\right)+h_{i j k}^{-1} d h_{i j k}+\alpha\left(g_{i j}\right)\left(a_{j k}\right)+a_{i j}=h_{i j k}^{-1} a_{i k} h_{i j k}
$$

Now let us consider some examples. Recall from Sect. 4.1 that bU(1) is 2-group with one morphism and $\mathrm{U}(1)$ as its group of 2-morphisms. A U(1) gerbe is principal bU(1)-2-bundle. Let's look at principal U(1)-bundles and then U(1) gerbes, to get a feel for how they are similar and how they differ.

To build a principal U(1)-bundle with a connection on it, we choose transition functions

$$
g_{i j}: U_{i} \cap U_{j} \rightarrow \mathrm{U}(1)
$$

such that

$$
g_{i j} g_{j k}=g_{i k}
$$

on each triple intersection. To put a connection on this bundle, we then choose a 1-form $A_{i}$ on each patch such that

$$
A_{i}=A_{j}+g_{i j} d g_{i j}^{-1}
$$

on each double intersection $U_{i} \cap U_{j}$. The curvature of this connection is then

$$
F_{i}=d A_{i}
$$

on the $i$ th patch. Note that $F_{i}=F_{j}$ on $U_{i} \cap U_{j}$, so we get a well-defined curvature 2-form $F$ on all of $M$.

To build a U(1) gerbe, we choose transition functions $h_{i j k}: U_{i} \cap U_{j} \cap U_{k} \rightarrow \mathrm{U}(1)$ such that

$$
h_{j k l} h_{i j l}=h_{i j k} h_{i k l}
$$

on each quadruple intersection. Remember, the 2-group bU(1) has only one morphism, the identity, so the transition functions $g_{i j}$ are trivial and can be ignored. To put a 2-connection on this gerbe, we must first choose a 2-form $B_{i}$ on each patch. Then we must choose a 1 -forms $a_{i j}$ on each double intersection. We require that

$$
B_{i}=B_{j}+d a_{i j}
$$


on each double intersection, and

$$
a_{i j}+a_{j k}=a_{i k}+h_{i j k} d h_{i j k}^{-1}
$$

on each triple intersection. The 2-curvature of this 2-connection is then

$$
Z_{i}=d B_{i}
$$

on the $i$ th patch. Note that $Z_{i}=Z_{j}$ on $U_{i} \cap U_{j}$, so we get a well-defined 2-curvature 3-form $Z$ on all of $M$.

There is a nice link between $\mathrm{U}(1)$ gerbes and cohomology, which in fact is the reason they were invented in the first place. For any principal U(1)-bundle with connection, the curvature $F$ is integral:

$$
\int_{\Sigma} F \in 2 \pi \mathbb{Z}
$$

for any closed surface $\Sigma$ mapped into $M$. In addition, $F$ is closed:

$$
d F=0 .
$$

Conversely, any closed, integral 2-form $F$ on $M$ is the curvature of some connection on a principal U(1)-bundle over $M$. Two different connections on the same bundle have curvature 2-forms that differ by an exact 2-form, so we get a well-defined element of the deRham cohomology $H^{2}(M, \mathbb{R})$ from a principal $\mathrm{U}(1)$-bundle. This idea can be refined further, and the upshot is that principal $\mathrm{U}(1)$-bundles over $M$ are classified by the cohomology group $H^{2}(M, \mathbb{Z})$.

Similarly, for any U(1) gerbe, the curvature 3 -form $Z$ is closed and integral, where the latter term now means that

$$
\int_{Y} Z \in 2 \pi \mathbb{Z}
$$

for any closed 3-manifold $Y$ mapped into $M$. Conversely, any such 3-form is the 2-curvature of some 2-connection on a U(1) gerbe over $M$-and in fact, U(1) gerbes over $M$ are classified by $H^{3}(M, \mathbb{Z})$.

This is just the beginning of a longer tale: namely, the story of characteristic classes in higher gauge theory $[19,83]$. Indeed, though higher gauge theory is only in its infancy, there is much more to say. But our story ends here. We invite the reader to go further.

Acknowledgments This paper is based on JH's notes of lectures given by JB at the 2nd School and Workshop on Quantum Gravity and Quantum Geometry, held as part of the 2010 Corfu Summer Institute. We thank George Zoupanos, Harald Grosse, and everyone else involved with the Corfu Summer Institute for making our stay a pleasant and productive one. We thank Urs Schreiber for many discussions of higher gauge theory, and thank Tim van Beek and David Roberts for catching some errors. This research was partially supported by an FQXi grant. 
Open Access This article is distributed under the terms of the Creative Commons Attribution Noncommercial License which permits any noncommercial use, distribution, and reproduction in any medium, provided the original author(s) and source are credited.

\section{References}

1. Aschieri, P., Cantini, L., Jurčo, B.: Nonabelian bundle gerbes, their differential geometry and gauge theory. Commun. Math. Phys. 254, 367-400 (2005). Also available as arXiv:hep-th/0312154

2. Aschieri, P., Jurčo, B.: Gerbes, M5-brane anomalies and $E_{8}$ gauge theory. JHEP 10, 068 (2004). Also available as arXiv:hep-th/0409200

3. Ashtekar, A., Lewandowski, J.: Differential geometry on the space of connections via graphs and projective limits. J. Geom. Phys. 17, 191-230 (1995). Also available as arXiv:hep-th/9412073

4. Baez, J.: Four-dimensional $B F$ theory as a topological quantum field theory. Lett. Math. Phys. 38, 129-143 (1996)

5. Baez, J.: Spin foam models. Class. Quantum Grav. 15, 1827-1858 (1998). Also available as arXiv: gr-qc/9709052

6. Baez, J.: An introduction to spin foam models of $B F$ theory and quantum gravity. In: Gausterer, H., Grosse, H. (eds.) Geometry and Quantum Physics, pp. 25-93. Springer, Berlin (2000)

7. Baez, J., Baratin, A., Freidel, L., Wise, D.: Infinite-dimensional representations of 2-groups. Available as arXiv:0812.4969

8. Baez, J., Crans, A.: Higher dimensional algebra VI: Lie 2-algebras. Theory Appl Categories 12, 492538 (2004). Also available as arXiv:math.0307263

9. Baez, J., Crans, A., Schreiber, U., Stevenson, D.: From loop groups to 2-groups. HHA 9, 101-135 (2007). Also available as arXiv:math.QA/0504123

10. Baez, J., Crans, A., Wise, D.: Exotic statistics for strings in $4 \mathrm{~d} B F$ theory. Adv. Theor. Math. Phys. 11, 707-749 (2007). Also available as arXiv:gr-qc/0603085

11. Baez, J., Hoffnung, A., Rogers, C.: Categorified symplectic geometry and the classical string. Commun. Math. Phys. 293, 701-715 (2010). Also available as arXiv:0808.0246

12. Baez, J., Huerta, J.: Division algebras and supersymmetry II. Available as arXiv:1003.3436

13. Baez, J., Lauda, A.: A prehistory of $n$-categorical physics. Available as arXiv:0908.2469

14. Baez, J., Lauda, A.: Higher dimensional algebra V: 2-groups. Theory Appl. Categories 12, 423-491 (2004). Also available as arXiv:math.0307200

15. Baez, J., Perez, A.: Quantization of strings and branes coupled to $B F$ theory. Adv. Theor. Math. Phys. 11, 1-19 (2007). Also available as arXiv:gr-qc/0605087

16. Baez, J., Rogers, C.: Categorified symplectic geometry and the string Lie 2-algebra. Homol. Homotopy Appl. (to appear). Available as arXiv:0901.4721

17. Baez, J., Schreiber, U.: Higher gauge theory. In: Davydov, A. et al. (eds.) Categories in Algebra, Geometry and Mathematical Physics. Contemp. Math., vol. 431, pp. 7-30. AMS, Providence (2007) Also available arXiv:math.0511710

18. Baez, J., Sawin, S.: Functional integration on spaces of connections. J. Funct. Analysis 150, 1-26 (1997). Also available as arXiv:q-alg/9507023

19. Baez, J., Stevenson, D.: The classifying space of a topological 2-group. In: Baas, N., Friedlander, E., Jahren, B., Østvær, P.A. (eds.) Algebraic Topology: The Abel Symposium 2007. Springer, Berlin (2009)

20. Bakovic, I., Jurčo, B.: The classifying topos of a topological bicategory. Available as arXiv:0902.1750

21. Balachandran, A.P., Lizzi, F., Sparano, G.: A new approach to strings and superstrings. Nucl. Phys. B 277, 359-387 (1986)

22. Baratin, A., Freidel, L.: Hidden quantum gravity in 3d Feynman diagrams. Class. Quant. Grav. 24, 1993-2026 (2007). Also available as arXiv:gr-qc/0604016

23. Baratin, A., Freidel, L.: Hidden quantum gravity in 4d Feynman diagrams: emergence of spin foams. Class. Quant. Grav. 24, 2027-2060 (2007). Also available as arXiv:hep-th/0611042

24. Baratin, A., Wise, D.: 2-Group representations for spin foams. Available as arXiv:0910.1542

25. Barrett, J.W., Mackaay, M.: Categorical representations of categorical groups. Theory Appl. Categories 16, 529-557 (2006). Also available as arXiv:math.0407463

26. Bartels, T.: Higher gauge theory: 2-bundles. Available as arXiv:math.CT/0410328 
27. Breen, L.: Notes on 1- and 2-gerbes. In: Baez, J., May, P. (eds.) Towards Higher Categories, pp. 193-235. Springer, Berlin (2009). Also available as arXiv:math.0611317

28. Breen, L.: Differential geometry of gerbes and differential forms. Available as arXiv:0802.1833

29. Breen, L., Messing, W.: Differential geometry of gerbes. Adv. Math. 198, 732-846 (2005). Available as arXiv:math.AG/0106083

30. Brylinski, J.-L.: Loop Spaces, Characteristic Classes and Geometric Quantization. Birkhäuser, Boston (1993)

31. Brylinski, J.-L.: Differentiable cohomology of gauge groups. Available as arXiv:math.DG/0011069

32. Brylinski, J.-L., McLaughlin, D.A.: The geometry of degree-four characteristic classes and of line bundles on loop spaces I. Duke Math. J. 75, 603-638 (1994)

33. Brylinski, J.-L., McLaughlin, D.A.: The geometry of degree-four characteristic classes and of line bundles on loop spaces II. Duke Math. J. 83, 105-139 (1996)

34. Carey, A.L., Johnson, S., Murray, M.K.: Holonomy on D-branes. J. Geom. Phys. 52, 186-216 (2004). Also available as arXiv:hep-th/0204199

35. Castellani, L., D’Auria, R., Fré, P.: Supergravity and Superstrings: A Geometric Perspective. World Scientific, Singapore (1991)

36. Crane, L., Kauffman, L., Yetter, D.: State-sum invariants of 4-manifolds I. Available as arXiv: hep-th/9409167

37. Crane, L., Sheppeard, M.D.: 2-Categorical Poincaré representations and state sum applications. Available as arXiv:math.0306440

38. Crane, L., Yetter, D.: A categorical construction of 4d TQFTs. In: Kauffman, L., Baadhio, R. (eds.) Quantum Topology, pp. 120-130. World Scientific, Singapore (1993). Also available as arXiv: hep-th/9301062

39. Crane, L., Yetter, D.N.: Measurable categories and 2-groups. Appl. Categorical Struct. 13, 501-516 (2005). Also available as arXiv:math.0305176

40. DeDonder, T.: Theorie Invariantive du Calcul des Variations. Gauthier-Villars, Paris (1935)

41. DeWitt-Morette, C., Maheshwari, A., Nelson, B.: Path integration in phase space. Gen. Relativ. Gravit. 8, 581-593 (1977)

42. Duff, M.J.: Supermembranes: the first fifteen weeks. Class. Quantum Grav. 5, 189-205 (1988). Also available at $\langle$ http://ccdb4fs.kek.jp/cgi-bin/img_index?8708425〉

43. Eckmann, B., Hilton, P.: Group-like structures in categories. Math. Ann. 145, 227-255 (1962)

44. Elgueta, J.: Representation theory of 2-groups on Kapranov and Voevodsky 2-vector spaces. Adv. Math. 213, 53-92 (2007). Also available as arXiv:math.0408120

45. Fairbairn, W., Perez, A.: Extended matter coupled to B F theory. Adv. Theor. Math. Phys. D78, 024013 (2008). Also available as arXiv:0709.4235

46. Fairbairn, W., Noui, K., Sardelli, F.: Canonical analysis of algebraic string actions. Available as arXiv:0908.0953

47. Forrester-Barker, M.: Group objects and internal categories. Available as arXiv:math.CT/0212065

48. Freed, D.S., Witten, E.: Anomalies in string theory with D-Branes, §6: Additional remarks. Asian J. Math. 3, 819-852 (1999). Also available as arXiv:hep-th/9907189

49. Freidel, L., Louapre, D.: Ponzano-Regge model revisited. I: Gauge fixing, observables and interacting spinning particles. Class. Quant. Grav. 21, 5685-5726 (2004). Also available as arXiv:hep-th/0401076

50. Freidel, L., Louapre, D.: Ponzano-Regge model revisited. II: Equivalence with Chern-Simons. Also available as arXiv:gr-qc/0410141

51. Freidel, L., Livine, E.: Ponzano-Regge model revisited. III: Feynman diagrams and effective field theory. Also available as arXiv:hep-th/0502106

52. Gawedzki, K.: Topological actions in two-dimensional quantum field theories. In: t'Hooft, G., Jaffe, A., Mack, G., Mitter, P.K., Stora, R. (eds.) Nonperturbative Quantum Field Theory, pp. 101-141. Plenum, New York (1988)

53. Gawedzki, K., Reis, N.: WZW branes and gerbes. Rev. Math. Phys. 14, 1281-1334 (2002). Also available as arXiv:hep-th/0205233

54. Getzler, E.: Lie theory for nilpotent $L_{\infty}$-algebras. Available as arXiv:math.0404003

55. Gotay, M., Isenberg, J., Marsden, J., Montgomery, R.: Momentum maps and classical relativistic fields. Part I: covariant field theory. Available as arXiv:physics/9801019

56. Girelli, F., Pfeiffer, H.: Higher gauge theory—differential versus integral formulation. J. Math. Phys. 45, 3949-3971 (2004). Also available as arXiv:hep-th/0309173 
57. Girelli, F., Pfeiffer, H., Popescu, E.M.: Topological higher gauge theory-from $B F$ to $B F C G$ theory. J. Math. Phys. 49, 032503 (2008). Also available as arXiv:0708.3051

58. Guillemin, V., Sternberg, S.: Symplectic Techniques in Physics. Cambridge University Press, Cambridge (1984)

59. Henriques, A.: Integrating $L_{\infty}$-algebras. Available as arXiv:math.0603563

60. Johnson, M.: The combinatorics of $n$-categorical pasting. J. Pure Appl. Algebra 62, 211-225 (1989)

61. Lack, S.: A 2-categories companion. In: Baez, J., May, P. (eds.) Towards Higher Categories, pp. 105191. Springer (2009). Also available as arXiv:math.0702535

62. Lerman, E.: Orbifolds as stacks? Available as arXiv:0806.4160

63. Lewandowski, J., Thiemann, T.: Diffeomorphism invariant quantum field theories of connections in terms of webs. Class. Quant. Grav. 16, 2299-2322 (1999). Also available as arXiv:gr-qc/9901015

64. Mac Lane, S., Whitehead, J.H.C.: On the 3-type of a complex. Proc. Nat. Acad. Sci. 36, 41-48 (1950)

65. Mackaay, M., Picken, R.: Holonomy and parallel transport for Abelian gerbes. Adv. Math. 170, 287339 (2002). Also available as arXiv:math.DG/0007053

66. Markl, M., Schnider, S., Stasheff, J.: Operads in Algebra, Topology and Physics. AMS, Providence, Rhode Island (2002)

67. Martins, J.F., Picken, R.: On two-dimensional holonomy. Available as arXiv:0710.4310

68. Martins, J.F., Picken, R.: A cubical set approach to 2-bundles with connection and Wilson surfaces. Available as arXiv:0808.3964

69. Martins, J.F., Picken, R.: The fundamental Gray 3-groupoid of a smooth manifold and local 3-dimensional holonomy based on a 2-crossed module. Available as arXiv:0907.2566

70. Milnor, J.: Remarks on infinite dimensional Lie groups. In: Relativity, Groups and Topology II, (Les Houches, 1983), North-Holland, Amsterdam (1984)

71. Moerdijk, I.: Introduction to the language of stacks and gerbes. Available as arXiv:math.AT/0212266

72. Montesinos, M., Perez, A.: Two-dimensional topological field theories coupled to four-dimensional BF theory. Phys. Rev. D77, 104020 (2008). Also available as arXiv:0709.4235

73. Murray, M.K.: Bundle gerbes. J. London Math. Soc. 54, 403-416 (1996). Also available as arXiv: dg-ga/9407015

74. Murray, M.K.: An introduction to bundle gerbes. Available as arXiv:0712.1651

75. Murray, M.K., Stevenson, D.: Higgs fields, bundle gerbes and string structures. Commun. Math. Phys. 243, 541-555 (2003). Also available as arXiv:math.DG/0106179

76. Pfeiffer, H.: Higher gauge theory and a non-Abelian generalization of 2-form electromagnetism. Ann. Phys. 308, 447-477 (2003). Also available as arXiv:hep-th/0304074

77. Power, A.J.: A 2-categorical pasting theorem. J. Algebra 129, 439-445 (1990)

78. Pressley, A., Segal, G.: Loop Groups. Oxford University Press, Oxford (1986)

79. Roberts, D., Schreiber, U.: The inner automorphism 3-group of a strict 2-group. Also available as arXiv:0708.1741

80. Rovelli, C.: Quantum Gravity. Cambridge University Press, Cambridge (2004). Also available at $\langle$ http:// www.cpt.univ-mrs.fr/ rovelli/book.pdf $\rangle$

81. Roytenberg, D.: On weak Lie 2-algebras. Available as arXiv:0712.3461

82. Sati, H.: Geometric and topological structures related to M-branes. Available as arXiv:1001.5020

83. Sati, H., Schreiber, U., Stasheff, J.: $L_{\infty}$-algebras and applications to string- and Chern-Simons $n$-transport. Available as arXiv:0801.3480

84. Schlessinger, M., Stasheff, J.: The Lie algebra structure of tangent cohomology and deformation theory. J. Pure Appl. Algebra 38, 313-322 (1985)

85. Schommer-Pries, C.: A finite-dimensional string 2-group. Available as arXiv:0911.2483

86. Schreiber, U.: Comments at the $n$-Category Café. Available at /http://golem.ph.utexas.edu/category/ 2009/09/questions_on_ncurvature.html>

87. Schreiber, U., Waldorf, K.: Parallel transport and functors. J. Homotopy Relat. Struct. 4, 187-244 (2009). Also available as arXiv:0705.0452

88. Schreiber, U., Waldorf, K.: Smooth functors vs. differential forms. Available as arXiv:0802.0663

89. Schreiber, U., Waldorf, K.: Connections on non-abelian gerbes and their holonomy. Available as arXiv:0808.1923

90. Stevenson, D.: The Geometry of Bundle Gerbes. Ph.D. thesis, University of Adelaide (2000). Also available as arXiv:math.DG/0004117 
91. Stolz, S., Teichner, P.: What is an elliptic object? In: Tillmann, U. (ed.) Topology, Geometry and Quantum Field Theory: Proceedings of the 2002 Oxford Symposium in Honour of the 60th Birthday of Graeme Segal. Cambridge Uuniversity Press, Cambridge (2004)

92. Waldorf, K.: String connections and Chern-Simons theory. Available as arXiv:0906.0117

93. Weyl, H.: Geodesic fields in the calculus of variation for multiple integrals. Ann. Math. 36, 607629 (1935)

94. Whitehead, J.H.C.: Note on a previous paper entitled 'On adding relations to homotopy groups'. Ann. Math. 47, 806-810 (1946)

95. Whitehead, J.H.C.: Combinatorial homotopy II. Bull. Am. Math. Soc. 55, 453-496 (1949)

96. Witten, E.: The index of the Dirac operator in loop space. In: Landweber, P.S. (ed.) Elliptic Curves and Modular Forms in Algebraic Topology. Lecture Notes in Mathematics, vol. 1326, pp. 161-181. Springer, Berlin (1988) 